\title{
DETECTION OF THE TRANSVERSE PROXIMITY EFFECT: RADIATIVE FEEDBACK FROM BRIGHT QSOs ${ }^{1}$
}

\author{
Thiago S. Gonçalves and Charles C. Steidel \\ California Institute of Technology, Mail Stop 105-24, Pasadena, CA 91125; tsg@astro.caltech.edu, ccs@astro.caltech.edu \\ AND \\ Max PetTini \\ Institute of Astronomy, Madingley Road, Cambridge CB3 OHA, UK; pettini@ast.cam.ac.uk \\ Received 2007 August 16; accepted 2007 November 20
}

\begin{abstract}
Measuring the response of the intergalactic medium (IGM) to a blast of ionizing radiation allows one to infer the physical properties of the medium and, in principle, the lifetime and isotropy of the radiating source. The most sensitive such measurements can be made if the source of radiation is near the line of sight to a bright background QSO. We present results based on deep Keck HIRES observations of the QSO triplet KP 76, KP 77, and KP 78 at $z \simeq 2.5$, with separations of $2^{\prime}-3^{\prime}$ on the plane of the sky. Using accurate systemic redshifts of the QSOs from near-IR spectroscopy, we quantify the state of the IGM gas in the proximity regions where the expected ionizing flux from the foreground QSOs exceeds that of the metagalactic background by factors of 10-200, assuming constant and isotropic emission. Based on the unusual ionization properties of the absorption systems with detected $\mathrm{H} \mathrm{I}, \mathrm{C}$ IV, and $\mathrm{O}$ VI, we conclude that the gas has been significantly affected by the UV radiation from the nearby QSOs. Aided by observations of the galaxy density near the foreground QSOs, we discuss several effects that may explain why the transverse proximity effect has eluded most previous attempts to detect it. Our observations suggest that the luminosities of KP 76 and KP 77 have remained comparable to current values over timescales of, respectively, $\Delta t>25 \mathrm{Myr}$ and $16 \mathrm{Myr}<\Delta t<33 \mathrm{Myr}$, consistent with typical QSO lifetimes estimated from independent, less direct methods. There is no evidence that the UV radiation from either QSO was significantly anisotropic during these intervals.
\end{abstract}

Subject headings: intergalactic medium — quasars: absorption lines — quasars: general

Online material: color figures

\section{INTRODUCTION}

The QSO "proximity effect" refers to the observation that the incidence of Ly $\alpha$ absorption from the intergalactic medium (IGM) decreases at redshifts close to that of the QSO. If this deficit is interpreted as being due to the influence of the radiation field of the QSO on the nearby IGM, then measurements of the $\mathrm{H}$ I density near QSOs of known luminosity may be used to determine the intensity of the metagalactic ionizing background (Murdoch et al. 1986; Bajtlik et al. 1988; Scott et al. 2000). The statistical significance of the QSO proximity effect is high enough that there is little doubt of its reality. However, the measurement of the intensity of the metagalactic radiation field from observations of the Ly $\alpha$ forest is subject to a number of systematic uncertainties, in particular the uncertainty in the QSO systemic redshift (e.g., Scott et al. 2000; Espey et al. 1989), the possibly overdense large-scale environment in which QSOs may be located (e.g., Faucher-Giguere et al. 2008), and the nonlinear response of the observed $\mathrm{H}$ I optical depth to changes in the radiation field intensity, such that only $\mathrm{H}$ I systems with relatively low $\mathrm{H}_{\mathrm{I}}$ optical depths $\left(\tau_{\mathrm{H}_{\mathrm{I}}} \lesssim 10\right)$ will be significantly altered. All of these effects lead to an overestimate of the background intensity through an underestimate of the sphere of influence of radiation from the QSO; QSO redshifts determined from the broad UV emission lines tend to be lower than the true systemic redshifts, so that the true "proximity region" where the QSO radiation field dominates over the background is actually

\footnotetext{
${ }^{1}$ Based on data obtained at the W. M. Keck Observatory, which is operated as a scientific partnership among the California Institute of Technology, the University of California, and NASA and made possible by the generous financial support of the W. M. Keck Foundation.
}

larger than inferred. Similarly, if QSOs are preferentially found in overdense regions, then the incidence of $\mathrm{H}$ I with sufficient optical depth to remain relatively unscathed by an enhanced radiation field will be higher than at an average location in the universe, suggesting that the QSO's effect on the local radiation field is smaller than it really is. Clearly, there are also statistical (as opposed to systematic) issues associated with the proximity effect, since one expects relatively large sample variance in the $\mathrm{H}$ I content between the relatively small volumes probed by individual QSOs.

The so-called transverse proximity effect (TPE), on the other hand, involves searching for the influence of foreground sources of UV radiation on the IGM absorption observed in the spectra of background objects. Unlike the line-of-sight proximity effect, measurements of the TPE can, in principle, constrain the radiative lifetime and the isotropy of the sources (e.g., Schirber et al. 2004). Line-of-sight measurements of the proximity effect are sensitive only to fluctuations in the source intensity on timescales of $\$ 10^{4}$ yr (e.g., Martini 2004), whereas transverse measurements can sample longer timescales - determined by the light travel time between the foreground source and the observed line of sightthat are more interesting in the context of QSO lifetimes. The sphere of influence of the radiation field from a source of UV photons, as for the line-of-sight proximity effect, depends on the luminosity of the source. For a typical bright QSO $(V \sim 18)$ at $z \sim 2.5$, the sphere within which an isotropically radiating QSO significantly dominates over the metagalactic background has a physical radius of $r \sim 5-10 \mathrm{Mpc}$, corresponding to a lineof-sight velocity range of $\Delta v_{r} \simeq 1250-2500 \mathrm{~km} \mathrm{~s}^{-1}$ for material moving with the Hubble expansion. The corresponding light 
travel time is $15-30 \mathrm{Myr}$. A number of quasi-independent arguments have suggested that bright QSOs typically have lifetimes of $10^{6}-10^{8}$ yr (see, e.g., Haehnelt et al. 1998; Richstone et al. 1998; Martini \& Weinberg 2001; Hosokawa 2002; Yu \& Tremaine 2002; Steidel et al. 2002), so that the potential sphere of influence of the UV radiation from bright QSOs and the timescale of QSO "events" may fortuitously be of the same order. The relevant angular scales are $\theta \lesssim 10^{\prime}$ on the sky at $z \sim 2-3$, with the amplitude of the expected effects varying as $1 / \theta^{2}$ and proportional to the (far-UV) luminosity of the sources.

A number of authors have used QSOs with small angular separations on the sky but at different redshifts to search for the TPE via observations of the Ly $\alpha$ forest (e.g., Fernández-Soto et al. 1995; Crotts \& Fang 1998; Croft 2004; Schirber et al. 2004). To date, as far as we are aware, no evidence for a reduction in the number of Ly $\alpha$ forest lines at redshifts near foreground QSOs has been found. ${ }^{2}$ In fact, all of the above-referenced works except Fernández-Soto et al. (1995) instead reported an excess of Ly $\alpha$ absorption systems at the redshifts where TPE deficits would have been expected. The common explanations for the lack of the expected signal include some combination of the QSO large-scale environment, short or intermittent radiative lifetimes of the QSOs, and anisotropy of the QSO radiation. More recently, Hennawi et al. (2006) found that high column density $\mathrm{H}$ I systems at the redshifts of foreground QSOs are much more common than associated systems of similarly high column density seen in the spectra of QSOs at their own redshifts. These authors also estimated that the clustering of the high column density $\mathrm{H}$ I systems with the QSOs may be even stronger than that of star-forming galaxies at the same redshifts. Such observations have been interpreted as evidence that $\mathrm{H}$ I gas is distributed anisotropically around QSOs, possibly because of QSO variability and/or anisotropic UV radiation. As a counterargument, however, observations of the $\mathrm{He}$ II, rather than $\mathrm{H} \mathrm{I}$, Ly $\alpha$ forest seem to show that foreground QSOs do in fact have a measurable effect on the ionization state of gas within volumes corresponding to light travel times of $\sim 10^{6}$ $10^{7}$ Myr (Jakobsen et al. 2003; Worseck \& Wisotzki 2006; Worseck et al. 2007). This may suggest that $\mathrm{H}$ I is not the most sensitive barometer of changes in the radiation field near QSOs. The key advantage of the $\mathrm{He}$ II transition is that it is sensitive to the intensity of high-energy photons that can plausibly only be produced by QSOs or AGNs, so that one can sense changes in both the intensity and the shape of the radiation field for local sources.

A phenomenon closely related to the TPE is the fluorescence of high column density $\mathrm{H}$ I gas in the vicinity of bright sources of ionizing photons (see, e.g., Gould \& Weinberg 1996; Cantalupo et al. 2005). Searches for fluorescence at the redshifts of QSOs have so far yielded mixed results. Adelberger et al. (2006) identified what appears to be a damped Ly $\alpha$ system (DLA) at $z=2.84$ that is producing $\operatorname{Ly} \alpha$ emission in response to the ionizing radiation from a very bright QSO located $\sim 380 \mathrm{kpc}$ away, implying that the QSO luminosity has remained approximately constant over the last $\sim 10^{6} \mathrm{yr}$ (and that the QSO is radiating isotropically). However, in another case, the lack of fluorescence around a QSO at a similar redshift was interpreted by Francis \& Bland-Hawthorn (2004) as evidence for anisotropic emission from the QSO.

Thus, the observational situation concerning the TPE is currently ambiguous. QSO lifetimes of $>10^{6} \mathrm{yr}$ and largely isotropic QSO radiation are supported by some observations but challenged

\footnotetext{
2 Gallerani et al. (2008) observe a "transmission gap" in the Ly $\alpha$ forest of a background $z=6.42 \mathrm{QSO}$ near the redshift of a foreground QSO at $z=5.65$, although the statistical significance of the region of reduced Ly $\alpha$ opacity is difficult to evaluate.
}

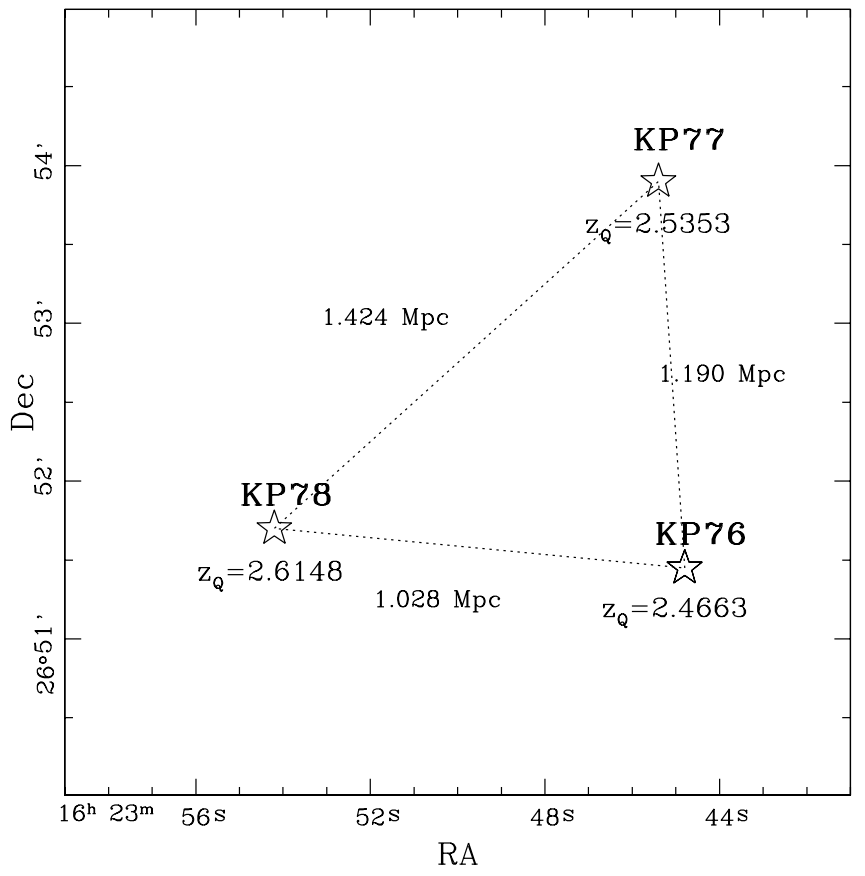

FIG. 1.-QSO KP 76, KP 77, and KP 78 triplet on the plane of the sky. The transverse physical distances between the QSO sight lines (evaluated at the redshift of the lower redshift QSO in each case) are indicated. The QSO systemic redshifts were measured from accurate near-IR spectroscopy $(\S 2.2)$.

by others. For this reason, we have elected to investigate the TPE using a different approach from that of most previous attempts. Rather than counting Ly $\alpha$ lines or evaluating the average Ly $\alpha$ opacity near foreground QSOs, we focus on the metal-line systems. Our strategy is to closely examine the ionization state of metal-line systems within the expected spheres of influence of foreground QSOs, using the combination of very high quality optical spectra of the background QSOs and precise determinations of the systemic redshifts of the foreground QSOs from near-IR spectroscopy. Our aim is to determine the strength of the ionizing radiation field seen by gas at various distances from the foreground QSOs, independently of the statistical limitations and systematic uncertainties inherent in line-counting or "mean flux" techniques. The hope is that the new data will help to explain why previous results have been so mixed, as well as better constrain the radiative lifetime and solid angle of QSO emission.

In the first stage of the program reported here, we focus on the QSO triplet KP 76, KP 77, and KP 78 (also known as Q1623+ 268). This grouping is among the best-studied in the literature, having figured prominently in earlier work aimed at measuring the coherence scale and clustering of the Ly $\alpha$ forest (e.g., Sargent et al. 1982; Crotts 1989; Crotts \& Fang 1998; Crotts et al. 1997). The relative configuration of the three QSOs is illustrated in Figures 1 and 2. Crotts \& Fang (1998) searched for the TPE in the Ly $\alpha$ forests of this triplet and concluded, based on counts of Ly $\alpha$ absorption lines, that there is no evidence that the foreground QSOs (KP 76 and KP 77) reduce the density of Ly $\alpha$ lines in the expected proximity regions. Rather, the line density appears to be higher than at an average location in the forest at comparable redshifts.

In this paper we present the highest quality high-resolution spectra of these QSOs to date, obtained with the recently upgraded High Resolution Echelle Spectrograph (HIRES) on the Keck I telescope. We have also obtained, as part of an ongoing survey of galaxies and the IGM at redshifts $1.8 \lesssim z \lesssim 3$ (see Steidel et al. 2004; Adelberger et al. 2005), spectra of approximately 300 other 


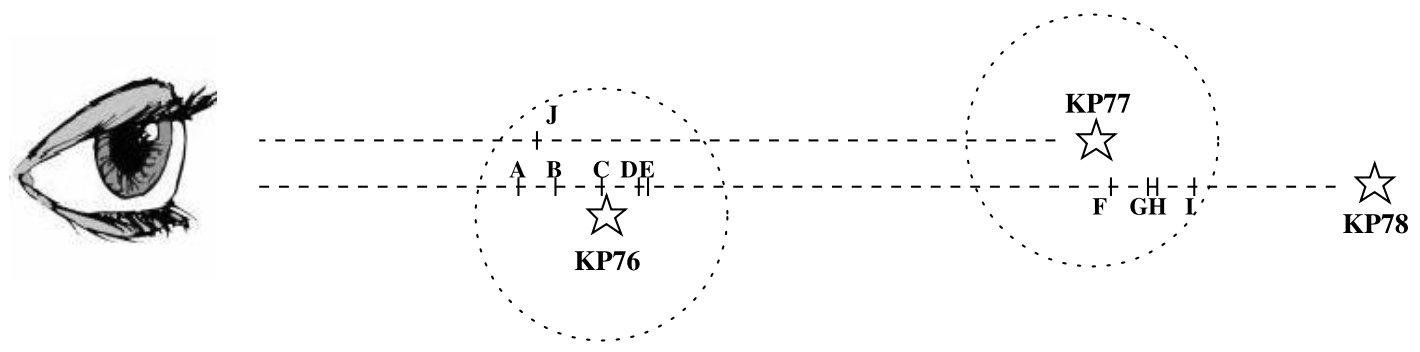

FIG. 2.-Schematic view of the QSO triplet. The relative positions of the metal-line absorption systems studied in this paper, labeled A-J, are indicated within dotted circles representing the proximity regions of the foreground QSOs KP 76 and KP 77. Within these volumes — spheres of physical radius $\sim 5 \mathrm{Mpc}-$ the ionizing flux from the nearby QSO is expected to exceed that due to the metagalactic background by more than 1 order of magnitude, if the QSOs radiate isotropically and their luminosities have remained approximately constant over the last 20-30 Myr. [See the electronic edition of the Journal for a color version of this figure.]

galaxies, QSOs, and AGNs in this redshift range within an $\sim 15^{\prime}$ field centered on the QSO triplet, providing unprecedented information on the surrounding large-scale structure. We examine the detailed properties of absorption-line systems within the expected proximity regions of the two foreground QSOs, and we interpret our findings in the context of our knowledge of the distribution of galaxies in the same regions.

\section{OBSERVATIONS}

\subsection{HIRES Spectroscopy}

The Q1623+2651 field (Sramek \& Weedman 1978), which includes the QSO triplet KP 76, KP 77, and KP 78, is part of a large-scale survey of galaxies and AGNs in the fields of bright QSOs that we have been conducting during the past several years (Steidel et al. 2004). The three QSOs have $u^{\prime}$-band magnitudes (measured from our photometry) of $18.62\left(\mathrm{KP} 76 ; z_{\mathrm{em}}=2.4663\right)$, $17.48\left(\mathrm{KP} 77 ; z_{\mathrm{em}}=2.5352\right)$, and $18.82\left(\mathrm{KP} 78 ; z_{\mathrm{em}}=2.6148\right)$, presenting a very unusual configuration of three bright QSOs at similar redshifts and within a few arcminutes on the sky. The angular separations between pairs are 127" (KP 76-KP 78), 147" (KP 76-KP 77), and 177" (KP 77-KP 78), corresponding to proper transverse distances (evaluated at the redshift of the lower redshift member of each pair) of $1.028,1.190$, and 1.424 physical Mpc, respectively, for the $\Omega_{M}=0.3, \Omega_{\Lambda}=0.7, H_{0}=$ $70 \mathrm{~km} \mathrm{~s}^{-1} \mathrm{Mpc}^{-1}(h=0.7)$ cosmology that is assumed throughout this paper (see Fig. 1).

The observations reported here were obtained on the nights of 2005 May 31, June 1, and October 9 and 10 with the Keck I telescope and HIRES (Vogt et al. 1994) using the UV cross-disperser and UV-sensitive CCD array. The data cover the wavelength range 3100-6000 $\AA$, with small gaps in spectral coverage near 4000 and $5000 \AA$ corresponding to gaps between CCD chips on the detector. All exposures were taken through the $1.148^{\prime \prime}$ slit, resulting in a resolution of $8.5 \mathrm{~km} \mathrm{~s}^{-1}$ (FWHM), sampled with $\sim 3$ pixels per resolution element. Some additional HIRES spectra of KP 76 and KP 77, obtained in 1995 August by W. Sargent and collaborators, were also included. The MAKEE data reduction package, written by T. Barlow, was used to process the twodimensional HIRES spectra, extract them, map them onto a vacuum heliocentric wavelength scale, and finally combine them; continuum fitting prior to the merging of echelle orders was accomplished using the IMANIP package, kindly provided by R. Simcoe. The final one-dimensional spectra have typical signalto-noise ratios $(\mathrm{S} / \mathrm{Ns})$ of $15-40$ per $2.8 \mathrm{~km} \mathrm{~s}^{-1}$ pixel.

\subsection{NIRSPEC Spectroscopy}

Given the importance of establishing the systemic redshifts of the QSOs, in order to accurately evaluate their effect on the nearby
IGM, we obtained near-IR spectra of all three QSOs encompassing the [O III] $\lambda \lambda 4959,5007$ and $\mathrm{H} \beta$ emission lines. The spectra were obtained in 2004 September with the Near Infrared Spectrograph (NIRSPEC; McLean et al. 1998) on the Keck II telescope in low-resolution $(R \simeq 1400$ ) mode using the NIRSPEC-5 filter (corresponding approximately to the near-IR $H$ band). The reductions were performed in the manner described in detail by Erb et al. (2006).

The redshifts of KP 76 and KP 77 were determined from Gaussian fits to the [O III] emission lines, yielding $z=2.4663 \pm$ 0.0003 and $2.5353 \pm 0.0003$, respectively. The redshift of KP 78, which is less crucial to our analysis since this QSO lies in the background of the other two, was measured to be $z=2.6148 \pm 0.0004$ from the $\mathrm{H} \beta$ line because [O III] falls in a region of very poor atmospheric transmission between the $H$ and $K$ bands. Note that published catalog redshifts for the three QSOs are 2.490, 2.5177, and 2.6017 (the last two from the Third Data Release of the Sloan Digital Sky Survey); they differ from the newly determined and more accurate systemic redshifts by $+2050,-1490$, and $-1090 \mathrm{~km} \mathrm{~s}^{-1}$, respectively. In our work, we have found that such large redshift offsets are in fact typical of bright QSOs, and it is worthwhile to point out that they are as large as or larger than the velocity range corresponding (for material moving with the Hubble flow) to distances over which the radiation field of a bright QSO dominates over the background ( $\operatorname{see} \S 3$ ). Thus, it is essential to determine accurate redshifts from near-IR spectroscopy for a meaningful assessment of the proximity effect.

Even so, the systematic uncertainties in the redshifts of the three QSOs are likely to exceed the statistical error of $\pm 30 \mathrm{~km} \mathrm{~s}^{-1}$ of our Gaussian fits. For example, Boroson (2005) has shown, using a large sample of low-redshift AGNs, that the redshifts defined by the [O III] doublet are lower than those of the host galaxies by $40 \mathrm{~km} \mathrm{~s}^{-1}$, on average, and that the offset can be up to 10 times higher in the most extreme cases. Nevertheless, the [O III] doublet is a far better indicator of systemic redshift than the broad UV emission lines commonly used. For the purposes of the present work, we assume that the above values are the systemic redshifts of the three QSOs considered here.

\section{IDENTIFICATION OF ABSORBERS AND ANALYSIS}

The first step in the analysis is to determine the wavelength intervals over which to search for evidence of the TPE in the HIRES spectra of the two background QSOs, KP 78 and KP 77. These intervals correspond to the physical distances to which the ionizing flux from the foreground QSOs will significantly dominate over the metagalactic radiation field. Given the difficulties experienced by previous observers in detecting the TPE, we decided to be deliberately conservative in the choice of interval and limited our analysis to the volume within which the expected radiation field 


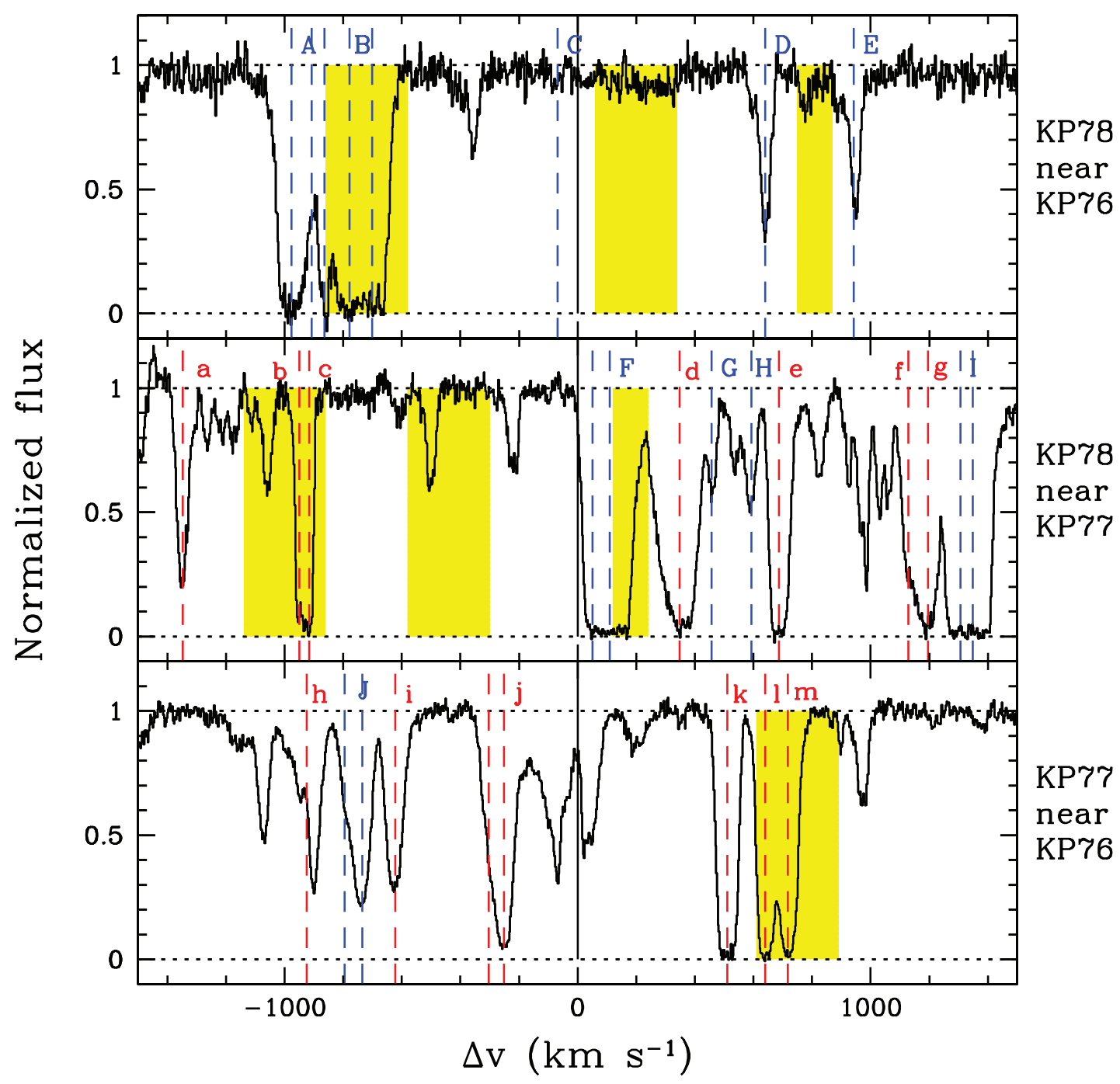

FIG. 3.-Portions of the spectra of the background QSOs near the redshifts of foreground QSOs. Each panel shows the Ly $\alpha$ forest within $\pm 1500 \mathrm{~km} \mathrm{~s}^{-1}$ of the foreground QSO redshift. The 10 metal-line systems identified within these proximity regions are denoted by letters A-J (blue dashed lines), while $13 \mathrm{H} \mathrm{I}-$ only absorbers with $\log N(\mathrm{H}$ I $)>13.5$ are indicated by lowercase letters (red dashed lines). The yellow regions mark the redshifts (together with their uncertainties) of seven spectroscopically confirmed galaxies within the proximity regions of KP 76 and KP 77 and close to the sight lines to KP 77 and KP 78. These galaxies are discussed in $\S 5.1$.

intensity from the proximate QSOs exceeds the metagalactic radiation field intensity by a factor of $\gtrsim 10$, where the impact on the IGM should be easiest to recognize. We calculated the QSO luminosities using their measured broadband $u^{\prime}$ fluxes (corresponding to $\mathrm{AB}$ magnitudes at rest-frame wavelengths of $\simeq 1000 \AA$, just above the Lyman limit at $912 \AA$ ); these values were corrected for line blanketing in the $\mathrm{Ly} \alpha$ forest estimated from the spectra themselves, increasing their brightness by $\simeq 20 \%$. We adopted a metagalactic background intensity at the Lyman limit of $J_{\mathrm{bg}}\left(\nu_{0}\right) \simeq$ $5 \times 10^{-22} \mathrm{ergs} \mathrm{s}^{-1} \mathrm{~cm}^{-2} \mathrm{~Hz}^{-1} \mathrm{sr}^{-1}$ (Scott et al. 2000; Tytler et al. 2004; Bolton et al. 2005). Using these numbers, the ionizing fluxes from KP 76 and KP 77 will exceed that from the metagalactic radiation field by factors of $\gtrsim 10$ within spheres of a physical radius of $\sim 5 \mathrm{Mpc}$, assuming that the QSOs radiate isotropically and that their luminosities have remained approximately constant over the relevant transverse light travel times of $\sim 10^{6}-10^{7} \mathrm{yr}$. In our cosmology, a radius of $5 \mathrm{Mpc}$ corresponds to a velocity interval $\Delta v \simeq$ $\pm 1250 \mathrm{~km} \mathrm{~s}^{-1}$ for material moving with the Hubble expansion. Considering the uncertainty in the systemic redshifts of the QSOs, which from the discussion above we estimate to be $\pm 100 \mathrm{~km} \mathrm{~s}^{-1}$, and an additional uncertainty of $\pm 200 \mathrm{~km} \mathrm{~s}^{-1}$ to account for departures from the Hubble flow due to the local density field, we settled on a velocity range $\Delta v \simeq \pm 1500 \mathrm{~km} \mathrm{~s}^{-1}$, centered on the systemic redshifts of KP 76 and KP 77, over which to examine the properties of the IGM in the HIRES spectra of KP 77 and KP 78. These "proximity regions" in the relevant portions of the Ly $\alpha$ forest are shown in Figure 3.

We identified metal-line systems within these proximity regions by the presence of Ly $\alpha$ absorption and either C IV $\lambda \lambda 1548$, 1550 or $\mathrm{O}$ vI $\lambda \lambda 1031,1037$ (or both). Since the first-order effect of an enhanced ionizing radiation field is to reduce the $\mathrm{H}_{\mathrm{I}}$ optical depth $\tau_{\mathrm{H}_{\mathrm{I}}}$, we did not require that strong $\mathrm{H}_{\mathrm{I}}$ be present; in fact, one system (system C below) has very weak $\mathrm{H}$ I that would not have been identified had the search been limited by Ly $\alpha$ optical depth. The wavelength coverage of the HIRES spectra down to $3100 \AA$ in the observed frame is important for confirming that a given absorption feature in the forest is indeed $\operatorname{Ly} \alpha$ (based on the presence of $\operatorname{Ly} \beta$ ) and for accurate measurement of the $\mathrm{H}$ i column density $N\left(\mathrm{H}_{\mathrm{I}}\right)$ in cases where the Ly $\alpha$ line is saturated (using higher order Lyman lines). Many of the Ly $\alpha$ lines within the proximity regions have no associated $\mathrm{C}$ IV or $\mathrm{O}$ vi absorption even at the sensitive detection limits reached by our HIRES spectra. In the subsequent analysis, we include all such $\mathrm{H} \mathrm{I}$-only systems with column densities $N(\mathrm{H} \mathrm{I})>10^{13.5} \mathrm{~cm}^{-2}$, for which we have 
TABLE 1

Metal-Line Systems in Proximity Regions

\begin{tabular}{|c|c|c|c|c|}
\hline System & Ion & $z$ & $\begin{array}{l}\log N \\
\left(\mathrm{~cm}^{-2}\right)\end{array}$ & $\begin{array}{c}b \\
\left(\mathrm{~km} \mathrm{~s}^{-1}\right)\end{array}$ \\
\hline \multirow[t]{4}{*}{ А } & H I & 2.4550 & $14.76 \pm 0.02$ & $31.4 \pm 0.8$ \\
\hline & $\mathrm{H} \mathrm{I}$ & 2.4558 & $13.36 \pm 0.13$ & $20.3 \pm 6.3$ \\
\hline & $\mathrm{O}$ vI & 2.4557 & $13.87 \pm 0.14$ & $41.8 \pm 15.0$ \\
\hline & C IV & 2.4550 & $12.92 \pm 0.04$ & $18.9 \pm 1.9$ \\
\hline \multirow[t]{8}{*}{ В } & $\mathrm{H} \mathrm{I}$ & 2.4563 & $14.12 \pm 0.03$ & $17.5 \pm 1.3$ \\
\hline & $\mathrm{H} \mathrm{I}_{\mathrm{I}}$ & 2.4573 & $14.93 \pm 0.04$ & $33.2 \pm 1.8$ \\
\hline & $\mathrm{H}_{\mathrm{I}}$ & 2.4582 & $14.83 \pm 0.03$ & $31.8 \pm 1.2$ \\
\hline & $\mathrm{O}$ vI & 2.4578 & $14.12 \pm 0.06$ & $28.5 \pm 4.5$ \\
\hline & $\mathrm{O} \mathrm{vI}$ & 2.4584 & $14.03 \pm 0.07$ & $20.5 \pm 3.4$ \\
\hline & C IV & 2.4564 & $12.87 \pm 0.04$ & $9.5 \pm 1.3$ \\
\hline & $\mathrm{C}$ IV & 2.4578 & $12.68 \pm 0.09$ & $8.5 \pm 2.9$ \\
\hline & $\mathrm{C}$ IV & 2.4583 & $12.85 \pm 0.07$ & $13.5 \pm 2.8$ \\
\hline \multirow[t]{3}{*}{$\mathrm{C}$} & $\mathrm{H}_{\mathrm{I}}$ & 2.4654 & $12.44 \pm 0.07$ & $23.9 \pm 4.8$ \\
\hline & $\mathrm{O}$ vI & 2.4656 & $14.62 \pm 0.02$ & $34.2 \pm 0.9$ \\
\hline & $\mathrm{C}$ IV & & $\leq 12.13$ & \\
\hline \multirow[t]{3}{*}{$\mathrm{D}$} & $\mathrm{H} \mathrm{I}$ & 2.4737 & $13.50 \pm 0.06$ & $23.1 \pm 3.1$ \\
\hline & $\mathrm{O}$ VI & 2.4738 & $13.53 \pm 0.17$ & $20.8 \pm 9.8$ \\
\hline & C Iv & 2.4736 & $12.83 \pm 0.05$ & $50.4 \pm 6.2$ \\
\hline \multirow[t]{3}{*}{ Е .......................... } & $\mathrm{H} \mathrm{I}_{\mathrm{I}}$ & 2.4772 & $13.41 \pm 0.05$ & $22.1 \pm 3.2$ \\
\hline & $\mathrm{O}$ VI & 2.4775 & $13.79 \pm 0.11$ & $23.8 \pm 6.1$ \\
\hline & $\mathrm{C}$ Iv & & $\leq 12.13$ & \\
\hline \multirow[t]{5}{*}{ 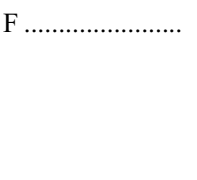 } & $\mathrm{H}$ I & 2.5358 & $14.24 \pm 0.89$ & $23.1 \pm 12.8$ \\
\hline & $\mathrm{H}_{\mathrm{I}}$ & 2.5365 & $15.26 \pm 0.16$ & $43.2 \pm 7.0$ \\
\hline & $\mathrm{O}$ VI & 2.5358 & $13.04 \pm 0.14$ & $8.4 \pm 3.7$ \\
\hline & $\mathrm{O} \mathrm{VI}$ & 2.5365 & $13.96 \pm 0.03$ & $36.6 \pm 3.3$ \\
\hline & C IV & 2.5363 & $\leq 12.28$ & 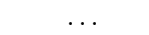 \\
\hline \multirow[t]{3}{*}{$\mathrm{G}$} & $\mathrm{H} \mathrm{I}$ & 2.5406 & $13.10 \pm 0.05$ & $17.8 \pm 1.3$ \\
\hline & $\mathrm{O}$ VI & 2.5407 & $13.47 \pm 0.07$ & $12.2 \pm 2.5$ \\
\hline & $\mathrm{C}$ Iv & 2.5406 & $12.42 \pm 0.06$ & $11.1 \pm 2.3$ \\
\hline \multirow[t]{3}{*}{ 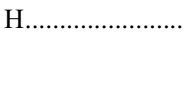 } & $\mathrm{H} \mathrm{I}$ & 2.5422 & $13.31 \pm 0.02$ & $22.8 \pm 1.1$ \\
\hline & $\mathrm{O}$ VI & 2.5422 & $13.62 \pm 0.06$ & $17.7 \pm 2.7$ \\
\hline & C Iv & 2.5422 & $12.46 \pm 0.07$ & $16.6 \pm 3.5$ \\
\hline \multirow[t]{6}{*}{ I } & $\mathrm{H} \mathrm{I}$ & 2.5506 & $15.62 \pm 0.91$ & $25.1 \pm 4.1$ \\
\hline & $\mathrm{H} \mathrm{I}_{\mathrm{I}}$ & 2.5511 & $15.64 \pm 0.64$ & $29.2 \pm 3.6$ \\
\hline & $\mathrm{O} \mathrm{VI}$ & 2.5506 & $13.37 \pm 0.13$ & $27.9 \pm 9.8$ \\
\hline & $\mathrm{O} \mathrm{VI}$ & 2.5510 & $12.97 \pm 0.27$ & $11.1 \pm 6.2$ \\
\hline & $\mathrm{C}$ Iv & 2.5507 & $13.07 \pm 0.03$ & $8.9 \pm 0.8$ \\
\hline & $\mathrm{C}$ IV & 2.5510 & $13.52 \pm 0.01$ & $16.6 \pm 0.6$ \\
\hline \multirow[t]{4}{*}{$\mathrm{J}$} & $\mathrm{H}_{\mathrm{I}}$ & 2.4571 & $13.07 \pm 0.05$ & $22.1 \pm 1.6$ \\
\hline & $\mathrm{H} \mathrm{I}_{\mathrm{I}}$ & 2.4578 & $13.85 \pm 0.01$ & $33.8 \pm 0.7$ \\
\hline & $\mathrm{O}$ VI & 2.4571 & $13.71 \pm 0.16$ & $5.7 \pm 3.2$ \\
\hline & C Iv & 2.4571 & $12.09 \pm 0.11$ & $10.9 \pm 3.8$ \\
\hline
\end{tabular}

confidence in both their identification and their measured column densities through the detection of higher order Lyman lines. In general, these $\mathrm{H}$ I-only systems do not provide significant constraints on the ionization level of the IGM, but they are included for completeness. The $3 \sigma$ upper limits to metal-line column densities are typically $\log N(\mathrm{O}$ vI $) \leq 12.7$ and $\log N(\mathrm{C}$ Iv $) \leq 12.1$ (with $N$ in units of $\mathrm{cm}^{-2}$ ).

We used the software package VPFIT ${ }^{3}$ to determine redshifts, column densities $N$, and Doppler parameters $b\left(\mathrm{~km} \mathrm{~s}^{-1}\right)$ for a total of 23 absorption systems. Table 1 lists the results of the profile fits for 10 systems with detected metal lines (labeled A-J), and Table 2 lists the results for $13 \mathrm{H} \mathrm{I}-$ only systems (labeled $a-m$ ). Portions of the spectra near selected transitions of interest, together with the VPFIT model fits, are reproduced in Figures 4-11. Again, we refer the reader to Figure 2 for a schematic illustration of the location of the various absorption systems along the two lines of

\footnotetext{
${ }^{3}$ See http://www.ast.cam.ac.uk/ rfc/vpfit.html.
}

TABLE 2

H i-Only Systems in Proximity Regions

\begin{tabular}{|c|c|c|c|}
\hline System & $z$ & $\begin{array}{c}\log N(\mathrm{H} \mathrm{I}) \\
\left(\mathrm{cm}^{-2}\right)\end{array}$ & $\begin{array}{c}b \\
\left(\mathrm{~km} \mathrm{~s}^{-1}\right)\end{array}$ \\
\hline a..... & 2.5193 & $13.65 \pm 0.02$ & $21.6 \pm 0.9$ \\
\hline$b$ & 2.5240 & $13.71 \pm 0.06$ & $15.7 \pm 1.4$ \\
\hline ..................... & 2.5244 & $13.74 \pm 0.05$ & $15.3 \pm 1.5$ \\
\hline$d \ldots \ldots \ldots \ldots \ldots \ldots \ldots$ & 2.5393 & $14.42 \pm 0.04$ & $56.8 \pm 2.8$ \\
\hline ............ & 2.5433 & $14.44 \pm 0.02$ & $25.4 \pm 0.4$ \\
\hline$f \ldots \ldots \ldots \ldots \ldots \ldots \ldots \ldots$ & 2.5485 & $13.63 \pm 0.05$ & $25.5 \pm 1.7$ \\
\hline$g$ & 2.5493 & $14.30 \pm 0.02$ & $36.0 \pm 1.7$ \\
\hline 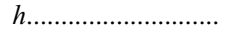 & 2.4556 & $13.56 \pm 0.02$ & $71.0 \pm 2.3$ \\
\hline$i$ & 2.4591 & $13.76 \pm 0.01$ & $33.1 \pm 0.5$ \\
\hline j & 2.4634 & $14.07 \pm 0.01$ & $29.4 \pm 0.6$ \\
\hline 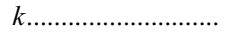 & 2.4722 & $14.44 \pm 0.01$ & $26.3 \pm 0.2$ \\
\hline$l$............................ & 2.4737 & $14.29 \pm 0.01$ & $24.0 \pm 0.3$ \\
\hline 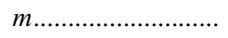 & 2.4746 & $14.21 \pm 0.01$ & $28.5 \pm 0.4$ \\
\hline
\end{tabular}

sight and to Figure 3 for an overall view of the Ly $\alpha$ forest within the proximity regions. We now briefly discuss each metal-line system in turn.

\subsection{Systems $A$ and $B(z=2.4554$ and 2.4573)}

These two systems are very close together, with a physical separation of less than $1 \mathrm{Mpc}$ (assuming negligible peculiar motions); each of them consists of multiple absorption components (see Fig. 4). Associated $\mathrm{C}$ IV and $\mathrm{O}$ VI absorption is detected in both $\mathrm{A}$ and $\mathrm{B}$. In the latter, there is a good match in velocity between $\mathrm{C}_{\text {IV }}$ and $\mathrm{O}$ vI in the two components at $z=2.4578$ and 2.4583 (at -735 and $-692 \mathrm{~km} \mathrm{~s}^{-1}$ in Fig. 4). At more negative velocities, however, there is a single broad $\mathrm{O}$ vi component approximately midway between the $\mathrm{C}$ iv redshifts $z=2.4550$ and 2.4564 , suggesting that this $\mathrm{O}$ vi absorption has an origin in diffuse, collisionally ionized gas, similar to the typical O VI systems discussed by Simcoe et al. (2002, hereafter S02).

$$
\text { 3.2. System } C(z=2.4655)
$$

This system is highly unusual in that it consists of a pair of very strong $\mathrm{O}$ vi lines with a very weak associated $\mathrm{Ly} \alpha$ line. No other metal lines are detected, including $\mathrm{N} v$ and $\mathrm{C}$ IV (see Fig. 5). It is thus reasonable to question whether our identification is correct. One complication is that the weaker member of the $\mathrm{O}$ vi doublet, $\lambda 1037$, is partially blended with a lower redshift Ly $\alpha$ line (see Fig. 6). However, VPFIT returns the same values of $z, \log N(\mathrm{O} \mathrm{vI})$, and $b$ irrespective of whether we fit the unblended $\mathrm{O}$ VI $\lambda 1031$ line on its own or simultaneously with the other member of the doublet and the blended Ly $\alpha$ line. In either case, VPFIT estimates the probability that the fit represents the data to be greater than $90 \%$. This absorption system is very close in velocity to the systemic redshift of KP $76\left(\Delta v=60 \mathrm{~km} \mathrm{~s}^{-1}\right)$. Its unusual properties, with strong $\mathrm{O}$ vi absorption, undetectable $\mathrm{C}$ Iv, and barely detectable $\operatorname{Ly} \alpha$ (at the $\mathrm{S} / \mathrm{N}$ of our data), are consistent with the gas being exposed to a greatly enhanced UV radiation field. We return to this point in $\S 4.2$.

\subsection{Systems $D$ and $E(z=2.4737$ and 2.4773)}

These two systems present clear and isolated Ly $\alpha$ absorption of moderate strength (see Fig. 7). We identify associated $\mathrm{O}$ vi absorption in both systems, although the $\mathrm{O}$ vi lines are located in a relatively crowded region of the spectrum. In particular, $\lambda 1037$ in system D is masked by a strong, saturated absorption feature (presumably Ly $\alpha$ at a lower redshift). C Iv is barely detected in system $\mathrm{D}$ and below our detection limit in system $\mathrm{E}$. 


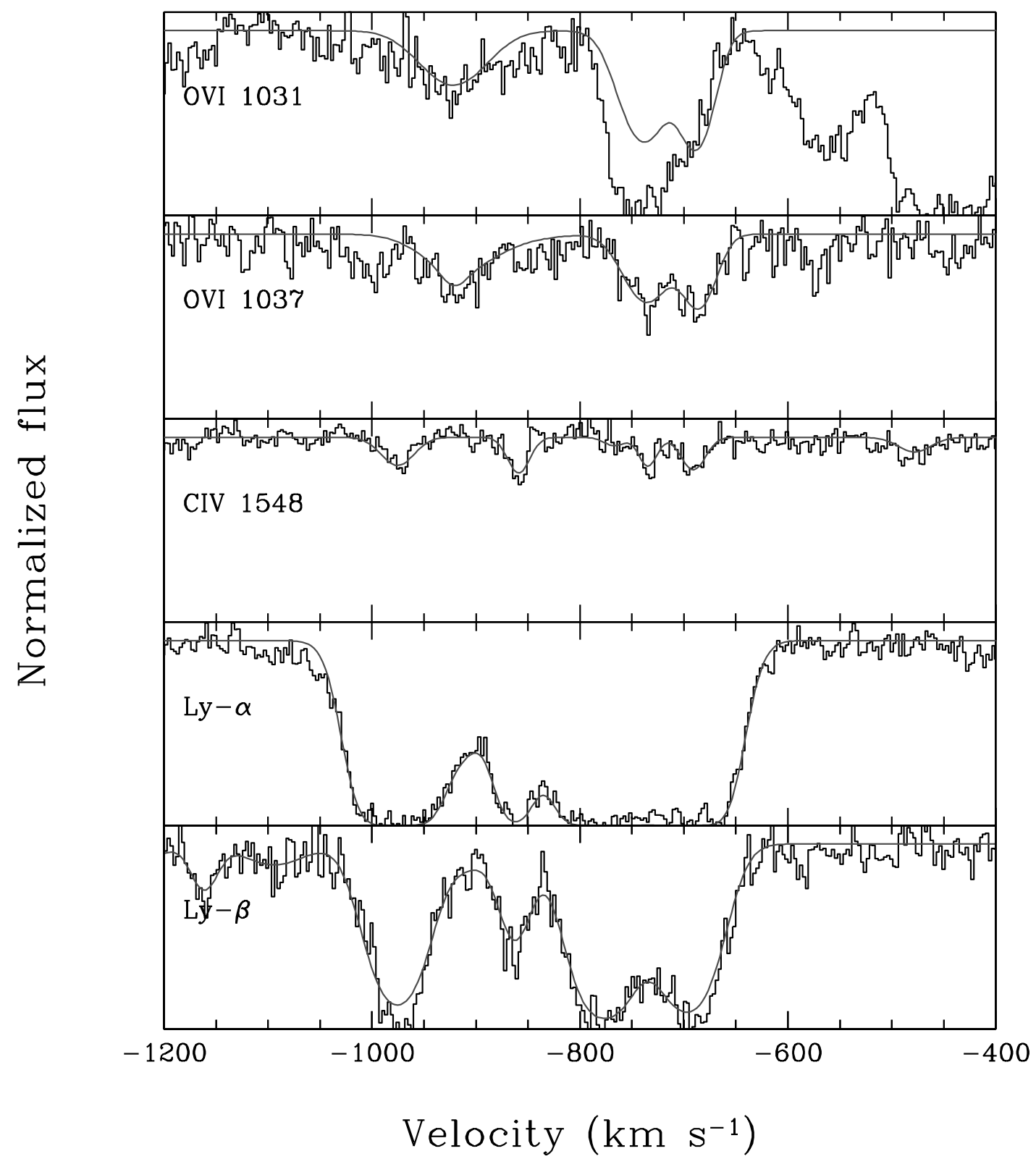

Fig. 4.- Spectra (histograms) and fitted Voigt profiles (solid lines) of selected absorption lines in systems A and B $(z=2.4554$ and 2.4573), as indicated. The $x$-axis velocity scale is relative to the systemic redshift of the foreground QSO, as in Fig. 3. [See the electronic edition of the Journal for a color version of this figure.]

\subsection{System $F(z=2.5362)$}

$\mathrm{H}$ I absorption is strong in this system, with $N(\mathrm{H} \mathrm{I})=2 \times$ $10^{15} \mathrm{~cm}^{-2}$, based on a strongly saturated Ly $\alpha$ line and higher Lyman series lines detected up to Ly $\delta$ (see Fig. 8). We clearly detect $\mathrm{O}$ vi $\lambda 1031$; although $\lambda 1037$ falls within a stronger feature (presumably Ly $\alpha$ at a lower redshift), the good redshift match of $\lambda 1031$ with the Lyman lines lends support to our identification. C Iv $\lambda 1548$ is very weak, below our detection limit. Again, this system is very close in redshift to the foreground QSO KP 77 (see Fig. 3); the velocity difference is only $\Delta v=+76 \mathrm{~km} \mathrm{~s}^{-1}$.

\subsection{Systems $G$ and $H(z=2.5406$ and 2.5422$)$}

These two systems, separated by only $\sim 150 \mathrm{~km} \mathrm{~s}^{-1}$, have very similar properties: weak Ly $\alpha$ and $\mathrm{C}$ IV but relatively strong $\mathrm{O}$ VI (see Fig. 9). Such properties are unusual for most metal-line absorption systems but consistent with a significant enhancement in the local ionizing radiation field.

\subsection{System I $(z=2.5508)$}

This system is the furthest from the foreground QSO among those considered here, with an inferred distance of $\sim 5 \mathrm{Mpc}$ from KP 77. Its properties are much more typical of intervening metalline systems, with relatively strong $\mathrm{C}$ IV and $\mathrm{H} \mathrm{I}$; its column density $N(\mathrm{H} \mathrm{I})=8.6 \times 10^{15} \mathrm{~cm}^{-2}$ is the largest among the absorbers in the present sample. Only the stronger member of the $\mathrm{O}$ vI doublet is detected ( $\lambda 1037$ is blended), but the proximity in velocity to $\mathrm{C}$ IV and $\mathrm{H}_{\mathrm{I}}$ supports the identification.

$$
\text { 3.7. System } J(z=2.4575)
$$

This is the only metal-line system in the proximity region of KP 76 probed by the line of sight to KP 77 (see Fig. 3). C Iv $\lambda 1548$ is marginally detected, while the $\mathrm{O}$ vi doublet lines are strong and narrow (see Fig. 11). Interestingly, the $b$ values of the $\mathrm{H}$ I $(z=$ 2.4571 component) and $\mathrm{O}$ vi lines ( $\mathrm{C}$ IV is too weak to provide a reliable constraint on $b$ ) scale approximately in proportion to the 


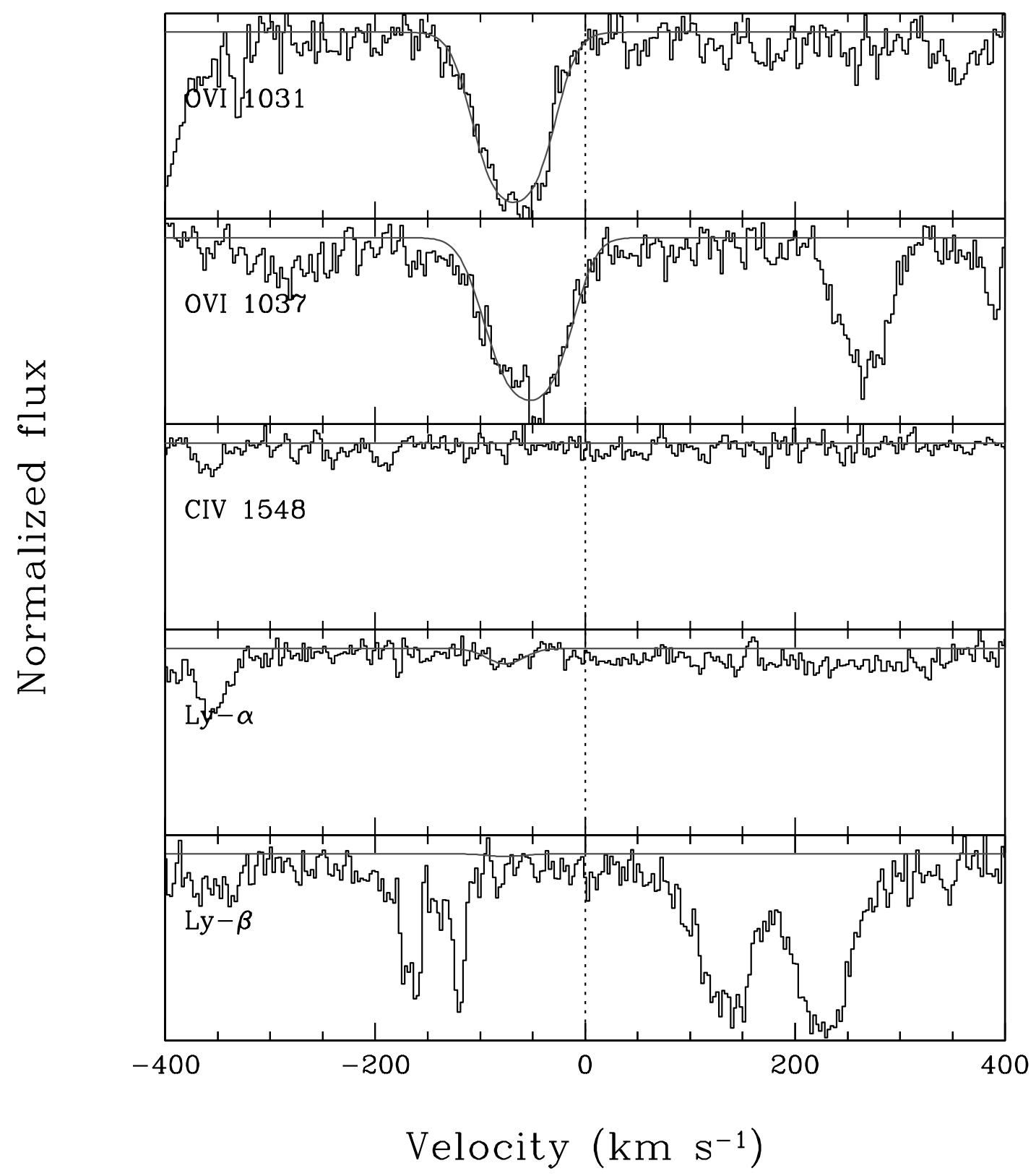

Fig. 5.-Same as Fig. 4, but for system C $(z=2.4655)$. There is strong absorption in $\mathrm{O}$ vi and a weak $\mathrm{H}$ I line. No other metal-absorption lines are detected. [See the electronic edition of the Journal for a color version of this figure.]

square root of the atomic mass, as expected from thermal broadening alone (i.e., with no contribution to the line widths by largescale turbulence). The implied temperature is $T \simeq 3 \times 10^{4} \mathrm{~K}$.

\section{THE EFFECTS OF THE QSO RADIATION FIELD}

\subsection{Inferences on the Radiation Field Intensity}

Most of the metal-line systems within the proximity regions of KP 76 and KP 77 exhibit strong $\mathrm{O}$ VI and relatively weak $\mathrm{C}$ IV and $\mathrm{H}$ I. In order to interpret these measurements quantitatively and assess the impact of the ionizing flux from KP 76 and KP 77 on their environments, we used the photoionization code CLOUDY (Ferland et al. 1998) to model the ionization state of the gas in systems A-J. The clouds were modeled as optically thin planeparallel slabs, illuminated on one side with a power-law radiation field ranging from 0.1 to 100 ryd. Hydrogen number density and metallicity were allowed to vary. Figure 12 shows the measured column density ratios (or limits, where applicable) together with the results of photoionization models for four values of the metal abundance, from solar to $1 / 1000$ of solar. The dashed lines show the line ratios expected for a given value of the dimensionless ionization parameter $U$, defined as

$$
U=\frac{\Phi}{n_{\mathrm{H}} c}=\frac{n_{\gamma}}{n_{\mathrm{H}}},
$$

where $\Phi$ is the flux of photons capable of ionizing $\mathrm{H}, n_{\gamma}$ is the ionizing photon number density, and $n_{\mathrm{H}}$ is hydrogen number density. We assume that the cloud is homogeneous so that the column density ratios are independent of cloud size.

From Figure 12 it can be readily appreciated that, for a given value of the ionization parameter, the ratio $N(\mathrm{O}$ vI $) / N(\mathrm{C}$ IV $)$ does not vary significantly with metallicity, provided of course that the $\mathrm{O} / \mathrm{C}$ ratio remains constant; in our modeling we have kept the relative abundance of the two elements at the solar value. Consequently, the measured column density ratios $N(\mathrm{O}$ vI $) / N(\mathrm{C}$ IV $)$ and 


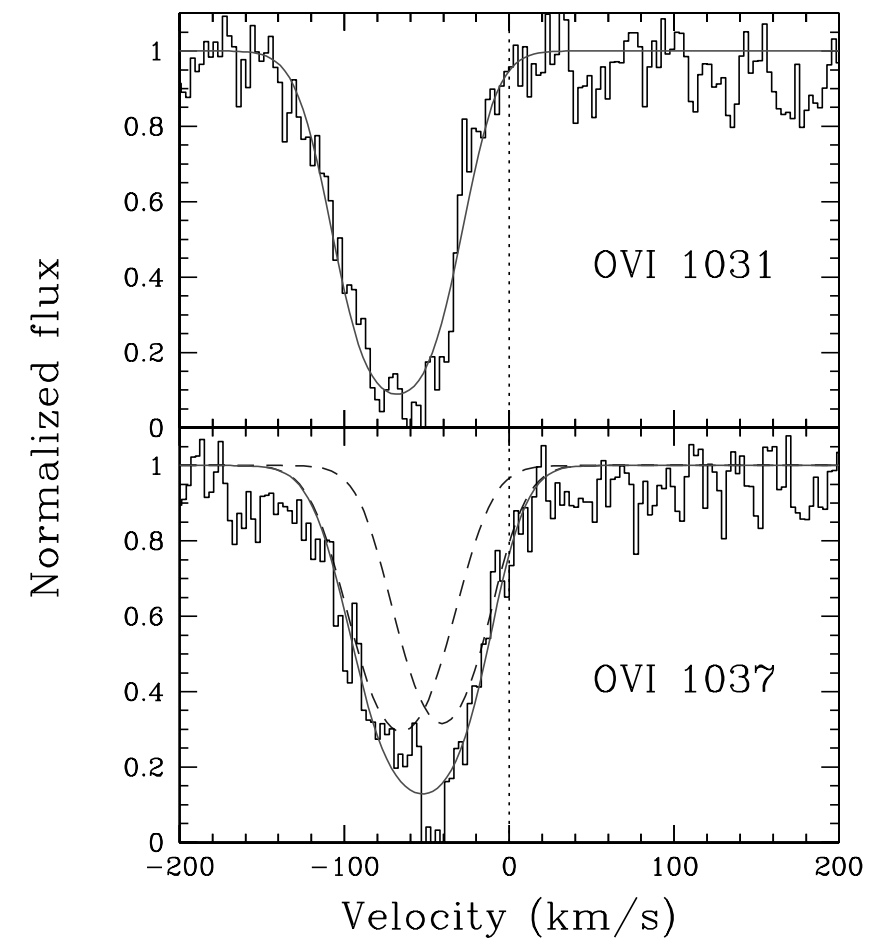

FIG. 6.- O vi absorption in system C (in the spectrum of KP 78 near the redshift of KP 76). As in the other figures, the histograms show the observed absorption profiles. In the top panel, the solid line is the synthetic absorption profile produced by VPFIT when fitting the $\mathrm{O}$ VI $\lambda 1031$ transition alone. The corresponding theoretical profile to the weaker member of the doublet is shown in the bottom panel (left dashed line). When combined with a lower redshift Ly $\alpha$ line (right dashed line) it produces a satisfactory fit to the blend (solid line). The velocity scale on the $x$-axis is the same as in Figs. 3 and 5. [See the electronic edition of the Journal for a color version of this figure.]

$N(\mathrm{O} v \mathrm{v}) / N(\mathrm{H} \mathrm{I})$ can be used to constrain the ionization parameter. Values of $\log U$ for each of the 10 metal-line systems are listed in column (8) of Table 3 and can be seen to be mostly between $\log U \simeq-1$ and $0 .{ }^{4}$ The typical uncertainties in $\log U$ given the assumed radiation field shape are $\sim 0.2-0.3$. The implied metallicities of the clouds, constrained by the ratios $N(\mathrm{O} v \mathrm{vI}) / N(\mathrm{H} \mathrm{I})$ and $N(\mathrm{C}$ IV $) / N(\mathrm{H} \mathrm{I})$, generally lie between a few tenths of solar and $1 / 1000$ of solar, values that are typical of Ly $\alpha$ forest clouds (e.g., Simcoe et al. 2004, hereafter S04). One exception is system C, which exhibits an unusually high column density of $\mathrm{O}$ VI compared to $\mathrm{H}$ I and $\mathrm{C}$ IV; its location on the diagram in Figure 12 cannot be reproduced even by the models with solar metallicity. Also shown in Figure 12 are points drawn from a combination of the samples of Bergeron et al. (2002, hereafter B02) and Carswell et al. (2002, hereafter C02), consisting of systems selected by $\mathrm{O}$ VI absorption only, without regard to $N(\mathrm{H} \mathrm{I})$. In general, nothing is known about the environment of the $\mathrm{O}$ vi systems in the B02+ C02 comparison sample, although one of the systems from B02 is within $1500 \mathrm{~km} \mathrm{~s}^{-1}$ of the published QSO emission redshift and has only a lower limit on $\log N(\mathrm{O} \mathrm{vI}) / N(\mathrm{C}$ IV $)$, implying that $\log U>-0.5$, similar to several of the proximate systems in our sample. We also note that there are two systems (out of 16) from the $\mathrm{B} 02+\mathrm{C} 02$ sample with implied metallicities similar to that of system $\mathrm{C}$, albeit with inferred values of $\log U$ that are lower

\footnotetext{
${ }^{4}$ For simplicity, here and in the following discussion we treat the upper limits to $N(\mathrm{C}$ IV $)$ as detections. Thus, the values of $U$ and $n_{\mathrm{H}}$ in Table 3 for systems $\mathrm{C}, \mathrm{E}$, and $\mathrm{F}$ are, respectively, lower and upper limits to the true values of the ionization parameter and hydrogen density.
}

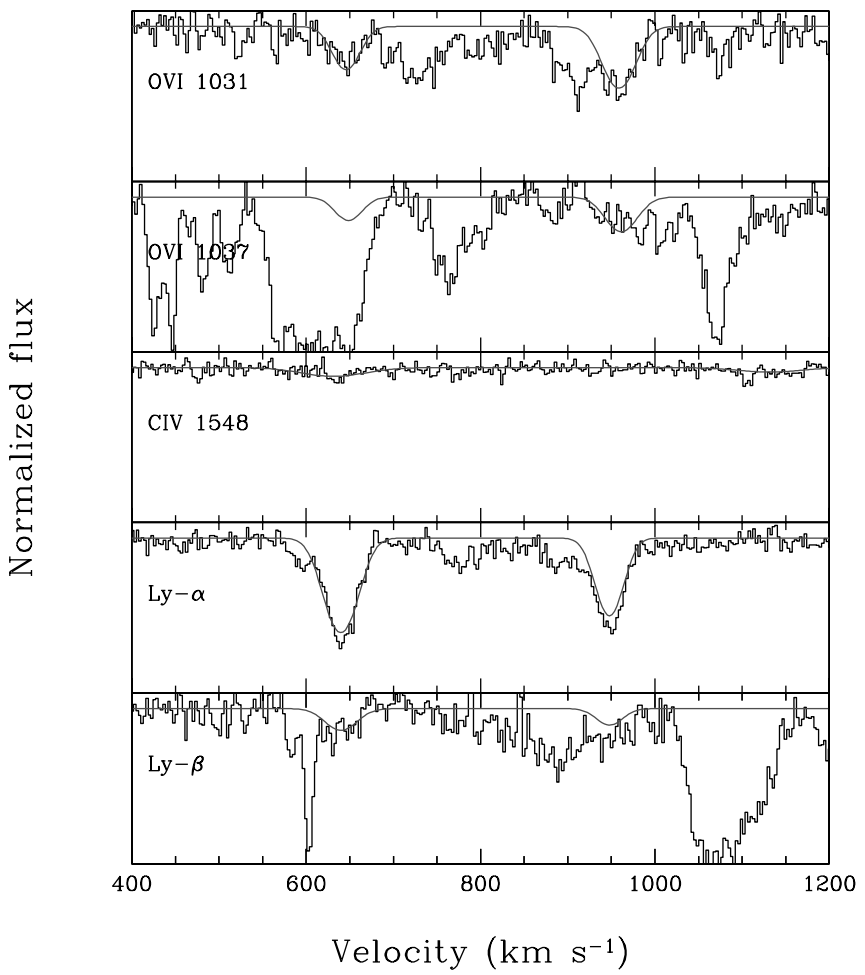

FIG. 7.-Same as Fig. 4, but for systems D and E $(z=2.4737$ and 2.4773). [See the electronic edition of the Journal for a color version of this figure.]

by $\sim 2$ dex. We return to a comparison of the properties of the proximate systems versus published "nonproximate" samples 5 below.

Having determined the value of $U$ for each metal-line system, we can now deduce the density $n_{\mathrm{H}}$ corresponding to a given value of $\Phi$ in equation (1). In particular, we are interested in examining the consequences of assuming that the ionizing flux seen by each cloud consists of the sum of two components: (1) the metagalactic ionizing background and (2) the radiation field of the QSO in the proximity zone of which the absorber is located. Specifically, we consider the scenario whereby the metagalactic background to which the cloud is exposed is boosted by the presence of the nearby QSO by a factor $g$, given by

$$
g=1+\frac{10^{-0.4\left(48.60+m_{912}\right)}}{(1+z) \pi J_{\mathrm{bg}}\left(\nu_{0}\right)}\left[\frac{d_{L}(z)}{r_{Q}}\right]^{2},
$$

where $d_{L}(z)$ is the luminosity distance to the QSO from Earth, and $J_{\mathrm{bg}}\left(\nu_{0}\right)$ is the background flux density at the Lyman limit; we adopt $J_{\mathrm{bg}}(\nu) \sim 5 \times 10^{-22}(\mathrm{~h} \nu / 13.6 \mathrm{ev})^{-1.8} \mathrm{ergs} \mathrm{s}^{-1} \mathrm{~cm}^{-2} \mathrm{sr}^{-1}$, which is consistent with the inferred hydrogen photoionization rate required to explain the mean Ly $\alpha$ forest optical depth (Bolton et al. 2005) and is approximately equal to the measured sum of the QSO and Lyman break galaxy contributions to the metagalactic radiation field at $z \sim 3$ (Shapley et al. 2006; Hunt et al. 2004). It is a factor of $\sim 2$ lower than the value of $J_{\nu}$ inferred from the QSO proximity effect (Scott et al. 2000) but is consistent within the uncertainties. Clearly, our results depend on the assumed intensity and spectral shape of the metagalactic radiation field, since we

\footnotetext{
5 We refer to these samples as "nonproximate," meaning "not known to be proximate." It is possible that some systems in the comparison samples are being ionized by more than the metagalactic radiation field.
} 


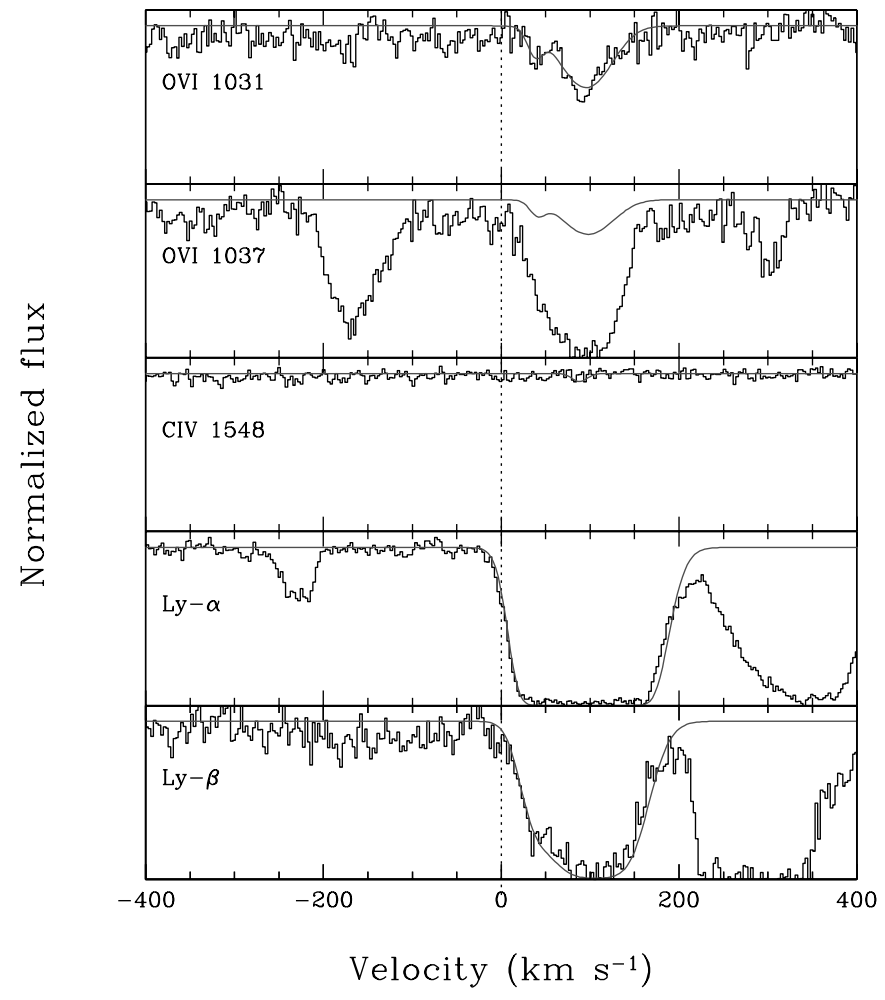

FIG. 8.- Same as Fig. 4, but for system F $(z=2.5362)$. Hydrogen absorption is strong, as is $\mathrm{O}$ VI $\lambda 1031$. $\mathrm{O}$ VI $\lambda 1037$ is blended with hydrogen lines at lower redshifts. C IV $\lambda 1548$, on the other hand, is very weak and below our detection limit. [See the electronic edition of the Journal for a color version of this figure.]

are seeking evidence for departures from these values produced by local sources of ionizing photons. The normalization is probably uncertain by at least a factor of $\sim 2$, so that inferred values of $g$ should be viewed as similarly uncertain. The value of $r_{Q}$ is the proper distance of the absorber from the nearby QSO, obtained by combining the transverse distance implied by their angular separation on the sky and the distance along the line of sight inferred from the redshift space velocity difference $\Delta v$ :

$$
r_{Q}=\left\{R^{2}(z)+\left[\frac{\Delta v}{H(z)}\right]^{2}\right\}^{1 / 2},
$$

where $R(z)$ is the proper distance on the plane of the sky between the foreground QSO and the sight line to the background QSO and $H(z)$ is the Hubble parameter; both quantities are evaluated at the redshift of the foreground QSO. As usual, the second term is affected by uncertainties due to departures from the Hubble flow caused by local density fields and by the systematic uncertainties in the QSO redshifts; together they may amount to less than $\sim 1 \mathrm{Mpc}\left(\simeq 260 \mathrm{~km} \mathrm{~s}^{-1}\right.$; Adelberger 2004). Values of $r_{Q}$ for all 23 absorption systems (metal-line systems and $\mathrm{H} \mathrm{I}-$ only systems) are given in column (5) of Table 3; given our choice of maximum value of $\Delta v(\operatorname{see} \S 3)$ and the QSO separations, they range from $\sim 1$ to $\sim 5 \mathrm{Mpc}$.

It is important to recall at this point that, as discussed in detail by Adelberger (2004), the geometry of the TPE is such that there is a time difference between, on the one hand, the time we measure the physical conditions of an absorbing cloud in the spectrum of a background QSO and, on the other hand, the time we measure the flux of the foreground QSO. The time difference $\Delta t$ depends on both the redshift and the transverse separation. For a velocity

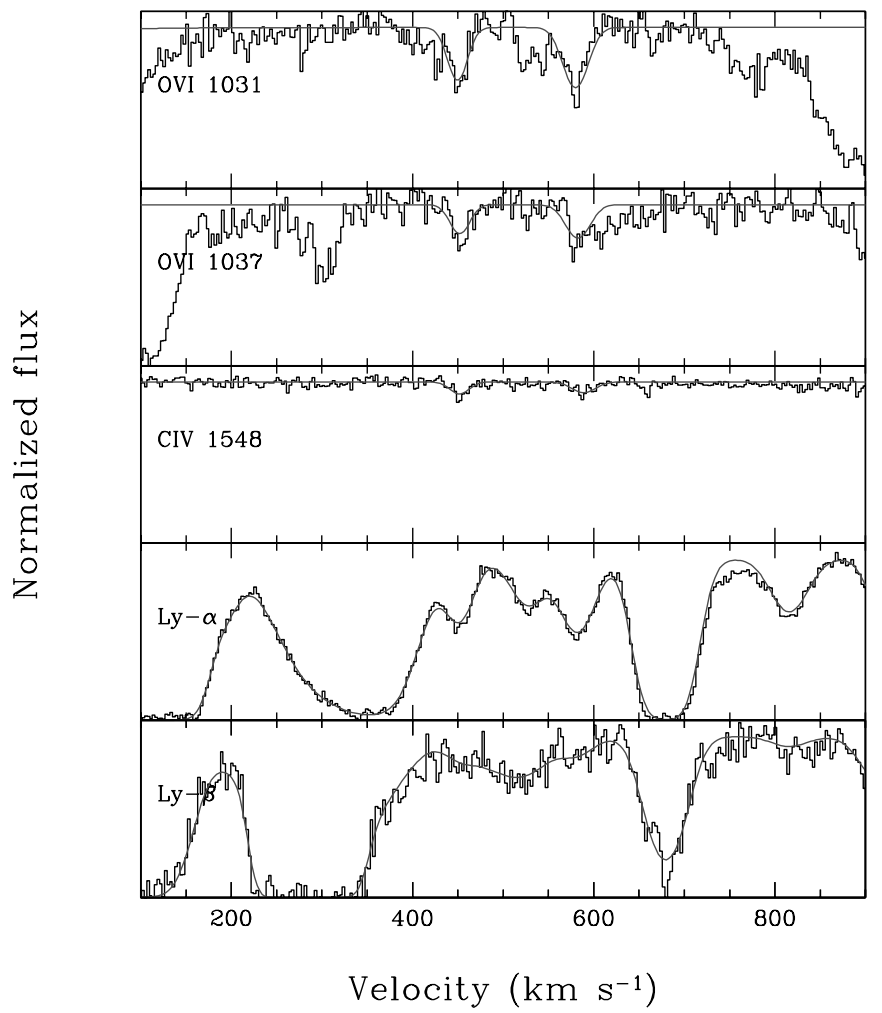

FIG. 9.- Same as Fig. 4, but for systems $\mathrm{G}$ and $\mathrm{H}(z=2.5406$ and 2.5422; $\mathrm{H}$ I-only systems $d$ and $e$ are also included in the plots, near 300 and $700 \mathrm{~km} \mathrm{~s}^{-1}$, respectively, as well as several weaker $\mathrm{Ly} \alpha$ components needed to fit the $\mathrm{H}$ i profiles; see Fig. 3). O vi lines are well defined and line up well with individual components within a complex system of $\mathrm{H}$ I absorption. As in the previous cases, C IV $\lambda 1548$ is weak. [See the electronic edition of the Journal for a color version of this figure.]

difference $\Delta v$ between the foreground QSO and an absorption system at proper separation $r_{Q}$,

$$
\Delta t=\frac{1}{c}\left[r_{Q}+\frac{\Delta v}{H(z)}\right] .
$$

Note that this time difference is not the same as the light travel time between the foreground QSO and the gas giving rise to the absorption, unless $z_{\mathrm{abs}}=z_{f_{Q}}$ (where $z_{f_{Q}}$ is the redshift of the foreground QSO). The value of $\Delta t$ for absorption systems with $\Delta v \gg 0$ (i.e., systems redshifted relative to the foreground QSO) will be nearly twice the light travel time $r_{Q} / c$, whereas $\Delta t<r_{Q} / c$ for systems with $\Delta v \ll 0$. In other words, absorption systems within the proximity region of a foreground QSO but located behind it (as viewed from Earth) probe physical conditions at times up to $\sim 30$ Myr earlier than the time we receive the QSO light on Earth. Conversely, systems in front of the QSO can provide a more recent measure at a given $r_{Q}$. Values of $\Delta t$ for all 23 systems considered in the two proximity regions of the foreground QSOs KP 76 and KP 77 are collected in column (6) of Table 3; they range between 0.2 and 33.4 Myr.

Returning to the QSO boost factors $g$ given by equation (2), their values, listed in column (7) of Table 3, were calculated under the assumption that the QSOs radiate isotropically and have maintained near-constant luminosity for the corresponding time intervals $\Delta t$. The lifetimes of bright QSOs are notoriously uncertain, but, as mentioned above, lifetimes of 1-100 Myr are believed to bracket the typical values. For the time being, we use our naive determinations of $g$ and examine the results for consistency with 


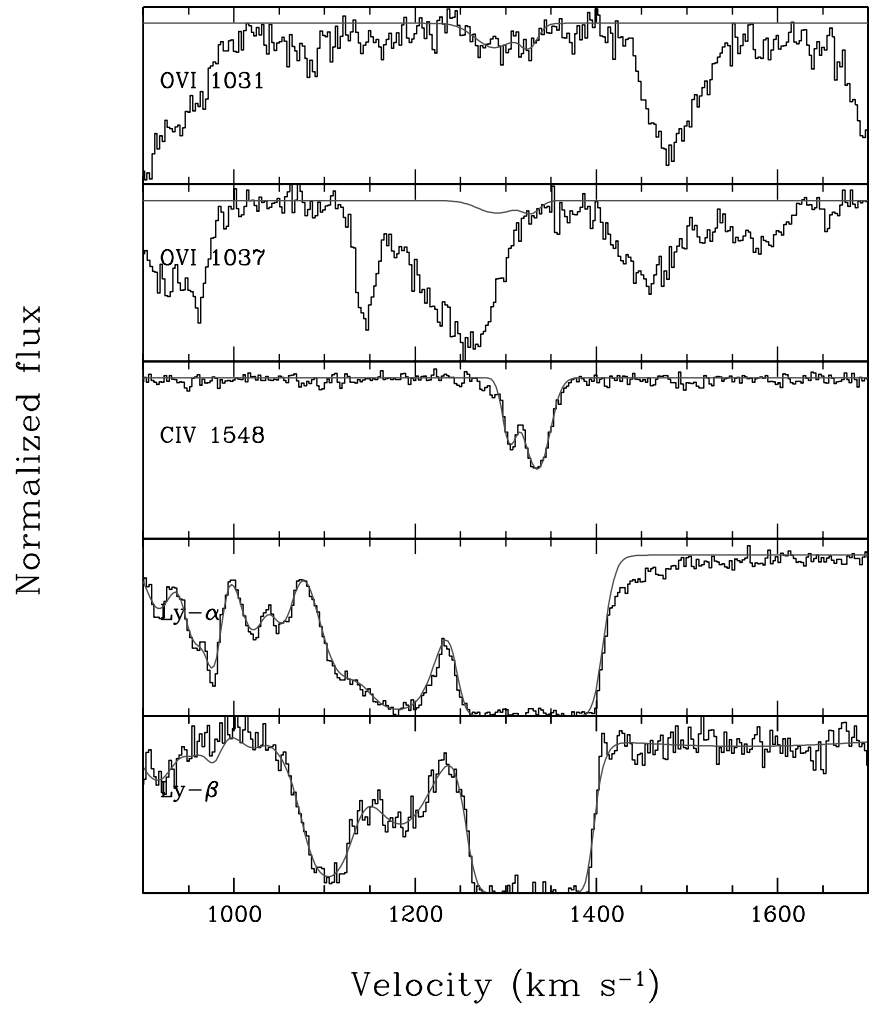

FIG. 10.- Same as Fig. 4, but for system I $(z=2.5508)$. Unlike the previous cases, $\mathrm{C}$ IV absorption is very well defined and stronger than $\mathrm{O}$ VI, as may be expected for a system near the edge of the proximity region. H I-only systems $f$ and $g$ appear near 1100 and $1200 \mathrm{~km} \mathrm{~s}^{-1}$, respectively (see Fig. 3). [See the electronic edition of the Journal for a color version of this figure.]

expectations. In particular, the calculated values of $g$ and $U$ lead, via equations (1) and (2), to the volume densities $n_{\mathrm{H}}$ listed in column (9) of Table 3; column (10) gives the corresponding overdensities relative to the mean density of baryons at $z=2.5$. From the table we see that the clouds probed in the proximity regions of KP 76 and KP 77 trace densities relative to the cosmic mean of $\log \rho / \rho_{0}=0.9-2.3$; these are typical values for metal-line systems of intermediate $\mathrm{H}$ I column density in the general IGM (e.g., S04).

With our photoionization modeling, we can further calculate the column densities of $\mathrm{H} \mathrm{I}, \mathrm{C}$ IV, and $\mathrm{O}$ VI that absorption systems A-J would produce, given their volume densities $n_{\mathrm{H}}$, in the absence of the radiation field from the nearby QSO. Using as input a radiation field now reduced by the boost factors $g$ given by equation (2), we obtained the column densities collected in Table 4. In Figures 13 and 14 we compare the ion column densities with and without the boost factors with the values measured in Ly $\alpha$ forest clouds by S04, supplemented by the smaller samples of B02 and C02 mentioned above. Unlike the S04 sample, which measured or placed limits on $N(\mathrm{H} \mathrm{I})$ and $N(\mathrm{O} \mathrm{VI})$ for all systems with $\log N(\mathrm{H} \mathrm{I})>13.6$, the latter studies searched for $\mathrm{O}$ vI detections independently of the associated $N(\mathrm{H} \mathrm{I})$, and thus may be more directly comparable to the sample of 10 proximate systems in which $\mathrm{O}$ VI is detected. Out of $16 \mathrm{O}$ vi systems in the combined B02 + C02 sample, two (12.5\%) have $\log N(\mathrm{H} \mathrm{I})<13.6$. Bergeron \& Herbert-Fort (2005) have presented a larger sample of O VI-selected systems, based on 10 QSO sight lines with column density sensitivity similar to $\mathrm{B} 02+\mathrm{C} 02$ and to the proximity regions in this paper. With a total survey redshift path of $\Delta z \simeq 2$, these authors find that 7 of $51(13.7 \%)$ of the O vi sys-

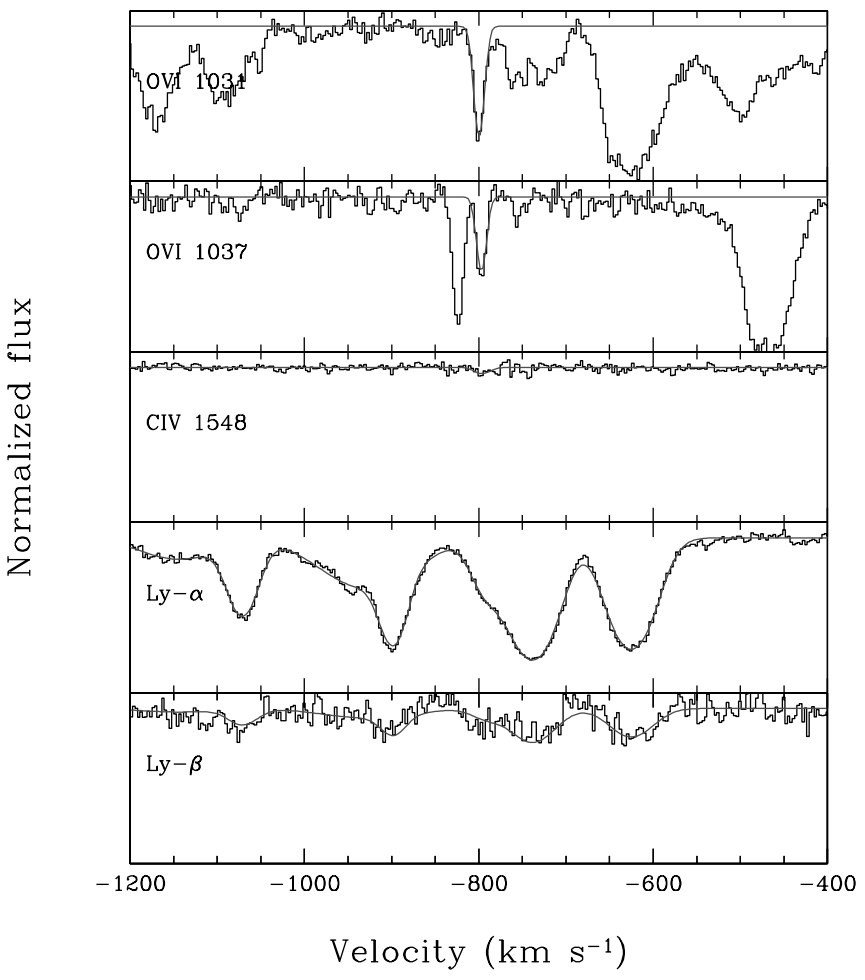

FIG. 11. - Same as Fig. 4, but for system $\mathrm{J}(z=2.4575)$. This system exhibits narrow $\mathrm{O}$ VI absorption lines; their width, when compared to that of the $\mathrm{H}$ I lines, indicates that they are formed in gas at $T \simeq 3 \times 10^{4} \mathrm{~K}$. The velocity range shown also includes $\mathrm{H}$ I- only systems $h$ and $i$ (see Fig. 3). [See the electronic edition of the Journal for a color version of this figure.]

tems at $z=2.3 \pm 0.3$ have $\log N(\mathrm{H} \mathrm{I})<13.6$. Of the $51 \mathrm{O}$ VI systems, all have detected lines of $\mathrm{C}$ IV with $\log N(\mathrm{C}$ IV $) \geq$ 12.30 (systems within $5000 \mathrm{~km} \mathrm{~s}^{-1}$ of the QSO redshifts were excluded from the sample). Note that, in contrast, 4 of the 10 proximate systems in our sample $(\mathrm{C}, \mathrm{E}, \mathrm{F}$, and $\mathrm{J})$ have $\log N(\mathrm{C}$ IV $)<$ 12.3. Although the actual column densities in the larger Bergeron \& Herbert-Fort (2005) sample are not yet published, the overall distribution of $N(\mathrm{O} \mathrm{vI}), N(\mathrm{C} \mathrm{IV})$, and $N(\mathrm{H} \mathrm{I})$ appear similar to the samples included in Figures 13 and 14.

Taking (for the moment) the observed properties of the proximate systems as shown in Figures 13 and 14, one sees that many of the systems lie simultaneously at the lower end of the distribution of $N(\mathrm{H} \mathrm{I})$ and the higher end of the distribution of $N(\mathrm{O} \mathrm{VI})$ among all systems with detected $\mathrm{O}$ vi. Only systems A, B, and $\mathrm{I}$ lie in regions inhabited by the main locus of general intervening systems; system $\mathrm{F}$, which is not anomalous in terms of its $N(\mathrm{O}$ vI) compared to $N(\mathrm{H}$ I $)$, has significantly lower $N(\mathrm{C}$ IV $)$ compared to any of the $\mathrm{S} 04$ systems with comparable $N(\mathrm{H} \mathrm{I})$ (Fig. 13). Systems C, D, E, G, and $\mathrm{H}$ all have $\log N(\mathrm{H} \mathrm{I})<13.6$, a trait we have seen is shared by only $\sim 13 \%$ of all systems with similar $N(\mathrm{O}$ vI $)$. Based on the statistics presented in Bergeron \& HerbertFort (2005), the expected number of such low $-N(\mathrm{H} \mathrm{I}) /$ strong$\mathrm{O}$ vi systems per unit redshift is $d n / d z \simeq 3.5$, whereas in the proximate regions we find five such systems within a total redshift window of only $\Delta z \simeq 0.10$ (or $d n / d z \simeq 50$ ). Figures 13 and 14 also include four $\mathrm{O}$ vI systems which $\mathrm{S} 02$ excluded from their analysis of strong $\mathrm{O}$ vI systems because the absorption redshifts are within $5000 \mathrm{~km} \mathrm{~s}^{-1}$ of the QSO emission redshifts. In two of the four cases, we have accurate redshift and luminosity information for the QSOs (Q1009+2956 and Q1700+6416), indicating that the two proximate absorbers have $\Delta v=-2760$ 


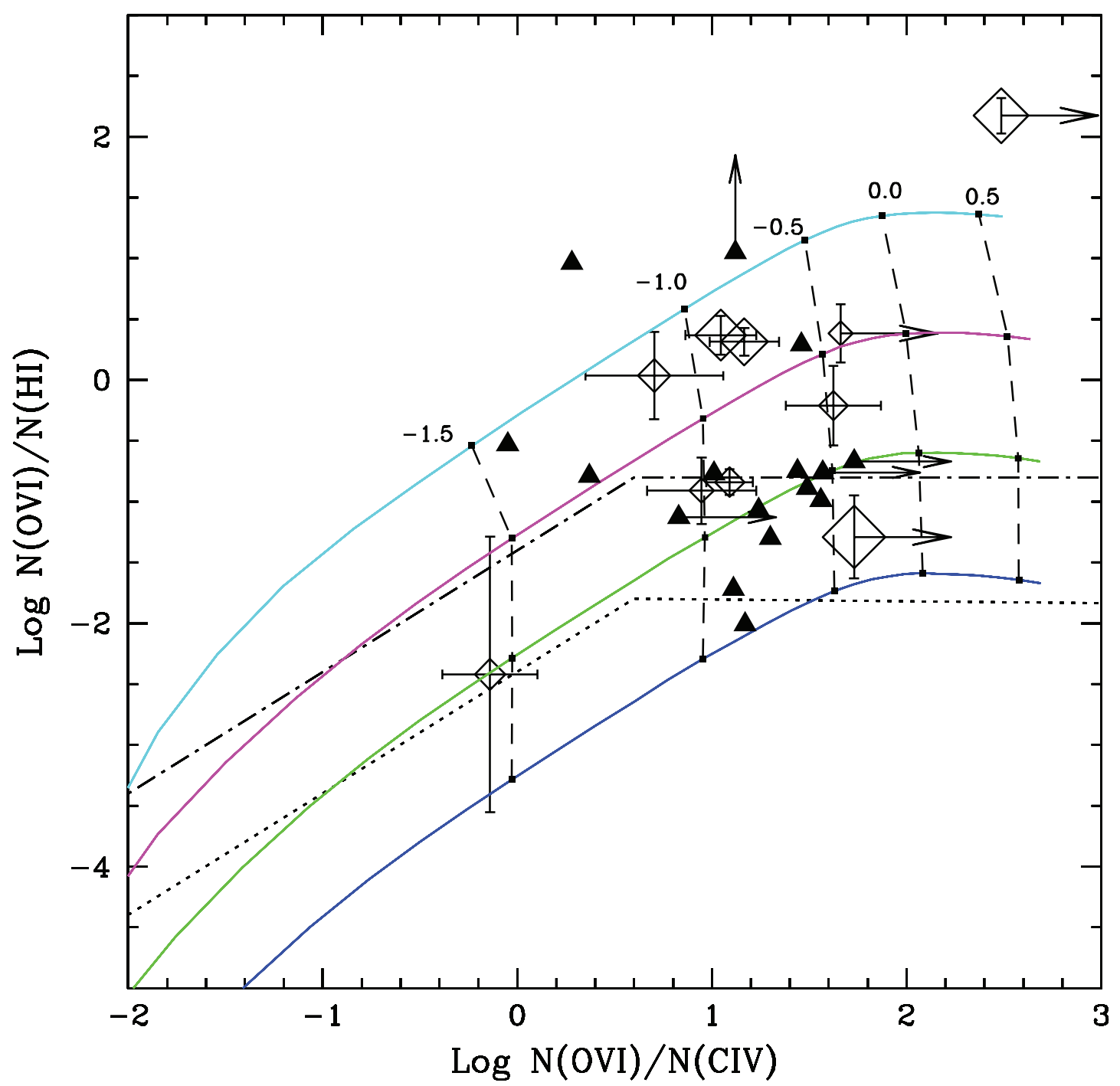

Fig. 12.-Open symbols indicate $N(\mathrm{O}$ vI $) / N(\mathrm{HI})$ vs. $N(\mathrm{O}$ vI $) / N(\mathrm{C}$ IV $)$ for metal-line systems in the proximity regions of $\mathrm{KP} 76$ and $\mathrm{KP} 77$. Symbol size is proportional to the logarithm of the boost factor $g$, listed in Table 3. Filled symbols indicate systems drawn from the O vI-selected samples of B02 and C02 for comparison (see text). Limits on ratios are indicated with arrows. Also shown are the ion ratios calculated with the photoionization code CLOUDY for an assumed power-law radiation field $J(\nu) \propto \nu^{-\alpha}$, with $\alpha=1.8$, a range of ionization parameters $U$, and four different values of metallicity, as follows: $[\mathrm{O} / \mathrm{H}]=-3($ dark blue $),[\mathrm{O} / \mathrm{H}]=-2($ green $),[\mathrm{O} / \mathrm{H}]=-1$ (magenta), and $[\mathrm{O} / \mathrm{H}]=0\left(\right.$ cyan), with the usual convention whereby $[\mathrm{O} / \mathrm{H}]=\log (\mathrm{O} / \mathrm{H})-\log (\mathrm{O} / \mathrm{H})_{\odot}$. Vertical dashed lines join the loci of constant ionization parameter $U$, with values as indicated. The dotted and dash-dotted lines refer to $\mathrm{H}$ I-only systems for which we determine upper $\operatorname{limits} \log N(\mathrm{O}$ vI $) \leq 12.7$ and $\log N(\mathrm{C}$ IV $) \leq 12.1 ;$ the dash-dotted line indicates systems with $\log N(\mathrm{H} \mathrm{I})=13.5$, while the dotted line indicates $\log N(\mathrm{H} \mathrm{I})=14.5$.

and $-600 \mathrm{~km} \mathrm{~s}^{-1}$ relative to the QSOs. Assuming negligible peculiar velocities, the corresponding line-of-sight physical separations are $\sim 10.6$ and $\sim 2.2 \mathrm{Mpc}$, with implied boost factors $g \sim 5$ and $\sim 120$, respectively. These two systems were the only O VI systems in the S02 sample which were not detected in C IV (see Fig. 13), and the latter system lies very close to systems $\mathrm{D}, \mathrm{E}, \mathrm{G}, \mathrm{H}$, and (especially) $\mathrm{J}$ in both $N(\mathrm{H} \mathrm{I})$ and $N(\mathrm{O} \mathrm{vI})$ (Fig. 14).

If the radiation from KP 76 and KP 77 is "switched off" (Figs. 13 and 14, red symbols), ${ }^{6}$ all of the proximate systems A-J are predicted to be detected in $\mathrm{C}$ IV and generally lie in the region occupied by less extreme intervening metal-line systems with intermediate $N\left(\mathrm{H}_{\mathrm{I}}\right)$. As seen in Figure 14, only 4 of the 10 proximate systems (A, B, E, and $\mathrm{J}$ ) are predicted to have detectable

\footnotetext{
${ }^{6}$ System F, which is only $1.4 \mathrm{Mpc}$ from KP 77, has such a high boost factor $(g \simeq 200)$ that it would give rise to a DLA in the absence of the radiation field from KP 77; to improve clarity we have not plotted this system for the "QSO off" case in Figs. 13 and 14.
}

$\mathrm{O}$ vI in the "QSO off" case. These numbers are consistent with the $N(\mathrm{H}$ I $)$-selected $\mathrm{S} 04$ sample; for example, in the range $14.0<$ $\log N(\mathrm{H} \mathrm{I})<16.0$, the $\mathrm{S} 04$ sample has 116 systems (an average redshift path density $d n / d z=56.6$ given the total path of the survey), of which $42 \%$ are not detected in $\mathrm{O}$ vI. In the total redshift path of the proximity regions considered here, $\Delta z \simeq 0.1$, one would then expect $n \simeq 5.6$ systems in the same range of $N(\mathrm{H} \mathrm{I})$ if it has an average density of such systems (see $\S 5$ ). Six of the proximate systems are predicted to lie in this range of $N(\mathrm{H} \mathrm{I})$ for the "QSO off" case: A, D, E, G, J, and H, of which three (A, E, and $J$ ) are predicted to yield $O$ vi detections. Similarly, the reduction of the assumed QSO-enhanced radiation field intensities also lowers the number of predicted $\mathrm{O}$ vI with $\log N(\mathrm{H} \mathrm{I})<13.6$ systems within the proximity regions from 5 to 0 , consistent with the expectation of only $n \simeq 0.35$ in $\Delta z \simeq 0.1$ from above.

In terms of the predictions for individual systems, most move to less extreme regions in both Figure 13 and Figure 14 in the "QSO off" case [including system F, which is not shown due to its large predicted $N(\mathrm{H} \mathrm{I})]$. The exceptions are systems $\mathrm{A}$ and $\mathrm{B}$, which lie inside the locus of points from the literature for either 
TABLE 3

Proximate Absorption System Model Results

\begin{tabular}{|c|c|c|c|c|c|c|c|c|c|}
\hline $\begin{array}{l}\text { System } \\
\text { (1) }\end{array}$ & $\begin{array}{c}z \\
(2)\end{array}$ & $\begin{array}{c}\mathrm{BQSO}^{\mathrm{a}} \\
\text { (3) }\end{array}$ & $\begin{array}{c}\mathrm{FQSO}^{\mathrm{b}} \\
(4)\end{array}$ & $\begin{array}{c}r_{Q}^{\mathrm{c}} \\
(\mathrm{Mpc}) \\
(5)\end{array}$ & $\begin{array}{c}\Delta t^{\mathrm{d}} \\
(\mathrm{Myr}) \\
(6)\end{array}$ & $\begin{array}{l}g^{\mathrm{e}} \\
(7)\end{array}$ & $\begin{array}{c}\log U^{\mathrm{f}} \\
\text { (8) }\end{array}$ & $\begin{array}{c}\log n_{H}{ }^{g} \\
\left(\mathrm{~cm}^{-3}\right) \\
(9)\end{array}$ & $\begin{array}{c}\log \rho / \rho_{0}{ }^{\mathrm{h}} \\
(z=2.5) \\
(10)\end{array}$ \\
\hline А & 2.4554 & KP 78 & KP 76 & 3.83 & 0.2 & 7.9 & -1.05 & -3.4 & 1.6 \\
\hline В & 2.4573 & KP 78 & KP 76 & 3.28 & 1.2 & 12.4 & -0.90 & -3.4 & 1.6 \\
\hline С & 2.4655 & KP 78 & KP 76 & 1.06 & 2.6 & 104.1 & +0.55 & -3.9 & 1.1 \\
\hline 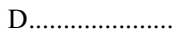 & 2.4737 & KP 78 & KP 76 & 2.69 & 16.8 & 16.1 & -1.20 & -3.0 & 2.0 \\
\hline E .......................... & 2.4773 & KP 78 & KP 76 & 3.86 & 24.5 & 7.8 & -0.35 & -4.1 & 0.9 \\
\hline F …….............. & 2.5362 & KP 78 & KP 77 & 1.38 & 5.5 & 196.0 & -0.35 & -2.7 & 2.3 \\
\hline G........................ & 2.5406 & KP 78 & KP 77 & 2.20 & 12.9 & 76.3 & -0.80 & -2.7 & 2.3 \\
\hline Н........................... & 2.5422 & KP 78 & KP 77 & 2.64 & 16.0 & 53.4 & -0.75 & -2.9 & 2.1 \\
\hline I & 2.5508 & KP 78 & KP 77 & 5.23 & 33.4 & 13.5 & -1.55 & -2.7 & 2.3 \\
\hline J & 2.4575 & KP 77 & KP 76 & 3.19 & 0.9 & 11.5 & -0.50 & -3.8 & 1.1 \\
\hline a & 2.5193 & KP 78 & KP 77 & 5.33 & 0.5 & 13.1 & $\ldots$ & $\ldots$ & $\ldots$ \\
\hline b...................... & 2.5240 & KP 78 & KP 77 & 3.87 & 0.8 & 24.8 & $\ldots$ & $\ldots$ & $\ldots$ \\
\hline$c$ & 2.5244 & KP 78 & KP 77 & 3.75 & 0.8 & 26.4 & $\ldots$ & $\ldots$ & $\ldots$ \\
\hline$d \ldots \ldots \ldots \ldots \ldots \ldots \ldots$ & 2.5393 & KP 78 & KP 77 & 1.89 & 10.5 & 104.2 & $\ldots$ & $\ldots$ & $\ldots$ \\
\hline e ........................... & 2.5433 & KP 78 & KP 77 & 2.95 & 18.1 & 42.7 & $\ldots$ & $\ldots$ & $\ldots$ \\
\hline$f \ldots \ldots \ldots \ldots \ldots \ldots \ldots$ & 2.5485 & KP 78 & KP 77 & 4.52 & 28.7 & 18.2 & $\ldots$ & $\ldots$ & $\ldots$ \\
\hline$g \ldots \ldots \ldots \ldots \ldots \ldots \ldots$ & 2.5493 & KP 78 & KP 77 & 4.76 & 30.3 & 16.3 & $\ldots$ & $\ldots$ & $\ldots$ \\
\hline h & 2.4556 & KP 77 & KP 76 & 3.82 & 0.8 & 8.0 & $\ldots$ & $\ldots$ & $\ldots$ \\
\hline$i$ & 2.4591 & KP 77 & KP 76 & 2.69 & 1.0 & 16.2 & $\ldots$ & $\ldots$ & $\ldots$ \\
\hline 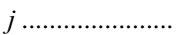 & 2.4634 & KP 77 & KP 76 & 1.49 & 1.7 & 52.9 & $\ldots$ & $\ldots$ & $\ldots$ \\
\hline k …… & 2.4722 & KP 77 & KP 76 & 2.30 & 13.9 & 22.2 & $\ldots$ & $\ldots$ & $\ldots$ \\
\hline$l \ldots \ldots \ldots \ldots \ldots \ldots$ & 2.4737 & KP 77 & KP 76 & 2.75 & 17.0 & 15.5 & $\ldots$ & $\ldots$ & $\ldots$ \\
\hline m...................... & 2.4746 & KP 77 & KP 76 & 3.04 & 18.9 & 12.7 & $\ldots$ & $\ldots$ & $\ldots$ \\
\hline
\end{tabular}

${ }^{\text {a }}$ Background QSO spectrum in which absorption is measured.

b Foreground QSO within "proximity region."

c Distance from foreground QSO.

d Difference between the time corresponding to the observed QSO luminosity and the time at which the physical conditions in the gas are being measured by the background QSO light (see text for discussion).

${ }^{\mathrm{e}}$ Enhancement of the ionizing radiation field under the assumption that the foreground QSO is radiating isotropically and had the same luminosity $\Delta t$ Myr ago.

${ }_{\mathrm{f}}^{\mathrm{f}}$ Inferred ionization parameter based on photoionization models.

${ }^{\mathrm{g}}$ Inferred hydrogen density, assuming that the radiation field intensity is produced by the foreground QSO and that it is a factor $g$ larger than the metagalactic background.

${ }_{\mathrm{h}}$ Hydrogen density in units of the mean density, assuming $\Omega_{b}=0.04$.

case, and possibly system I. The latter system, located at what we have considered to be the "edge" of the QSO proximity region (at a distance of $5.23 \mathrm{Mpc}$ from KP 77), exhibits $N(\mathrm{C}$ Iv $) / N(\mathrm{H} \mathrm{I})$ and $N(\mathrm{O} \mathrm{vI}) / N(\mathrm{H} \mathrm{I})$ ratios which may be more in line with those typically encountered in nonproximate systems and actually appears slightly anomalous in both ratios when the contribution of

TABLE 4

Column Densities with Reduced Radiation Field Intensities

\begin{tabular}{|c|c|c|c|}
\hline System & $N\left(\mathrm{H} \mathrm{I}_{\mathrm{I}}\right.$ & $N(\mathrm{O}$ vI $)$ & $N(\mathrm{C}$ IV $)$ \\
\hline А & 15.78 & 12.77 & 13.71 \\
\hline В & 16.39 & 13.09 & 14.13 \\
\hline …………........ & 13.63 & 12.35 & 13.58 \\
\hline 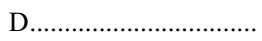 & 14.81 & 11.40 & 13.28 \\
\hline Е & 14.37 & 13.70 & 13.18 \\
\hline F & 20.08 & 10.10 & 13.01 \\
\hline . . & 15.01 & 10.32 & 12.86 \\
\hline . & 15.11 & 11.16 & 13.20 \\
\hline I & 17.21 & 10.68 & 13.38 \\
\hline J J & 15.11 & 13.22 & 13.32 \\
\hline
\end{tabular}

Note.-Column densities of $\mathrm{H} \mathrm{I}, \mathrm{O}$ vI, and $\mathrm{C}$ IV assuming the same physical densities as in Table 3, but with the ionization parameter reduced by the boost factor $g$ in each case.
KP 77 to the ionization rate is removed (see Figs. 13 and 14). It may be significant that this system, at the largest $\Delta t$ among those considered here, reflects the QSO UV luminosity 33 Myr prior to the time at which we observe KP 77 . We return to this point in $\S 6$.

In summary, none of the individual proximate metal-line systems is such an extreme outlier [as compared to samples with similar $N(\mathrm{H} \mathrm{I})$ or $N(\mathrm{O} \mathrm{vI})$ from the literature] that explaining the observations requires an enhanced radiation field from a nearby QSO. However, taken together, several statistical anomalies among the proximate sample are eliminated when the systems are modeled using radiation field intensities reduced by the $g$-factors in Table 3.

\subsection{Plausibility of Enhanced Radiation Field Intensities}

To summarize the main conclusions of the analysis above, we have shown with the aid of photoionization models that the high degree of ionization exhibited by most absorption systems within the proximity regions of KP 76 and KP 77 can be explained in terms of an enhanced radiation field and typical cloud densities (and metallicities). The enhancements in the ionizing flux to which the clouds are exposed are consistent with the values calculated from the observed luminosities of the two QSOs at the Lyman limit and the distances of the clouds from the nearby QSO, ignoring the possibility that the QSO far-UV luminosities may have varied 


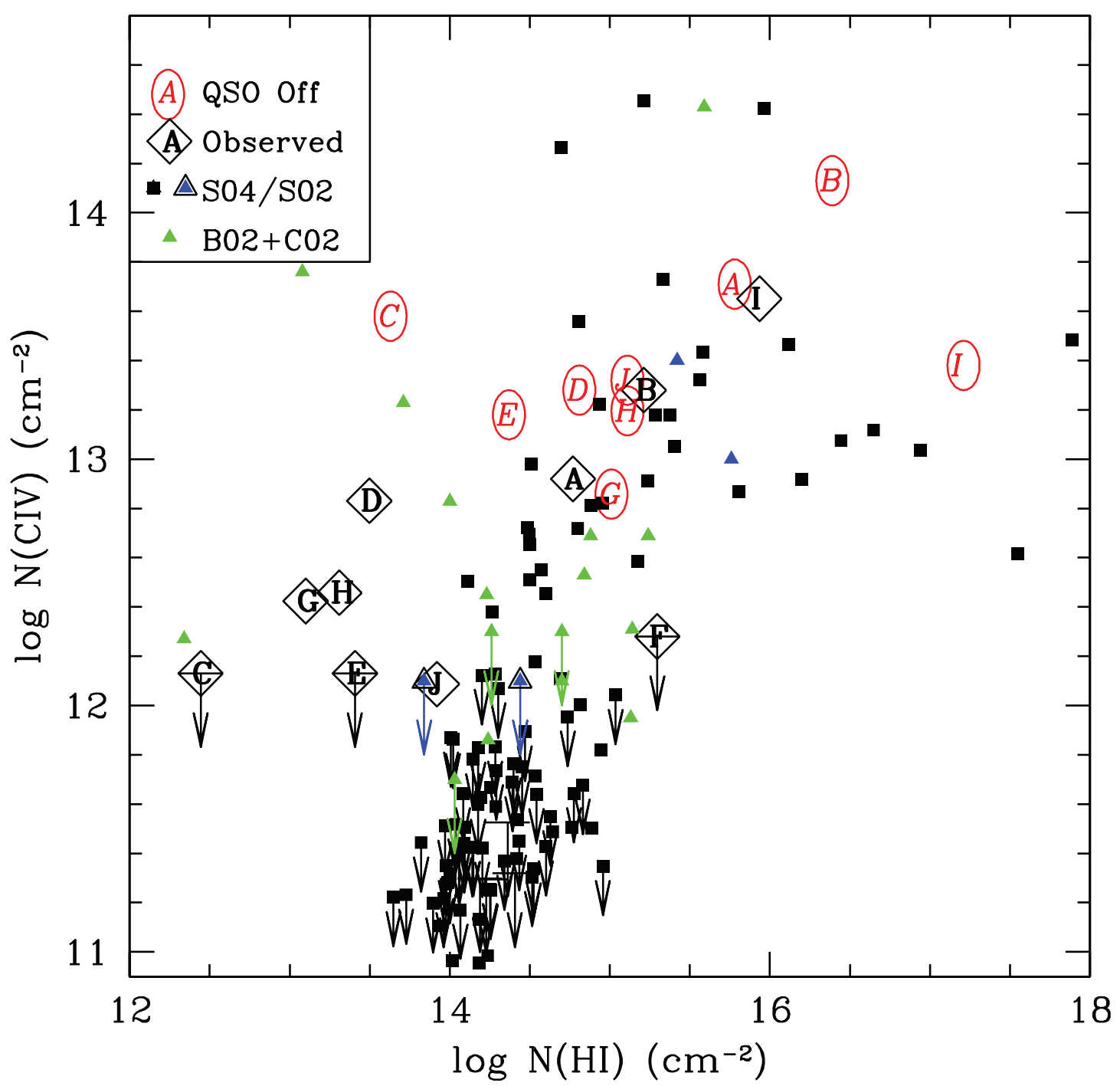

Fig. 13. - Comparison between nonproximate absorption-line systems from $\mathrm{S} 04, \mathrm{~B} 02$, and $\mathrm{C} 02$ and the proximate systems considered in this paper. The members of the $\mathrm{B} 02+\mathrm{C} 02$ sample (green triangles) were identified as $\mathrm{O}$ VI systems without regard to their $N(\mathrm{HI})$, whereas the $\mathrm{S} 04$ sample measured all systems with log $N(\mathrm{H} \mathrm{I})>13.6$. Upper limits on column densities are indicated with arrows. Diamonds labeled with the proximate system names are plotted at the measured values of column density, while red ellipses indicate the values which we calculate we would obtain if the incident radiation field were decreased by the boost factors $g$ listed in Table 3. Blue triangles indicate four systems from S02 that lie within $5000 \mathrm{~km} \mathrm{~s}^{-1}$ of the published QSO emission redshifts; the two outlined blue triangles indicate systems for which we have accurate QSO redshifts. The blue triangle near the observed system J corresponds to a proximate system near Q1700+64 with an estimated $g \simeq 120$ (see text).

significantly over the last $\sim 10^{7} \mathrm{yr}$ and that their emission may not be isotropic. In this section we examine more carefully a number of factors which may affect this interpretation.

First of all, we consider whether it is plausible that the relatively high values of the ionization parameter indicated by the observed ion ratios may result from unusually low densities $n_{\mathrm{H}}$ rather than abnormally high values of $n_{\gamma}$ (refer to eq. [1]). The densities so implied can be obtained straightforwardly by dividing the values of $n_{\mathrm{H}}$ and $\rho / \rho_{0}$ in columns (9) and (10) of Table 3 by the boost factors in column (7). In this scenario all of the clouds except one (system I) would be at the most mild overdensities relative to the cosmic mean; in the most extreme cases (systems C, E, and F) the absorption lines we see would arise in gas with a density lower than the cosmic mean (i.e., in voids). As pointed out by S02, such underdensities would imply proper sizes in excess of $1 \mathrm{Mpc}$; in such circumstances it is difficult to understand how the $\mathrm{O}$ vi absorption lines would remain so narrow, with $b$ values of $25-35 \mathrm{~km} \mathrm{~s}^{-1}$, when the differential Hubble flow across such large volumes would exceed $v_{\mathrm{H}} \simeq 250 \mathrm{~km} \mathrm{~s}^{-1}$. We thus consider it more likely, given the presence of nearby bright QSOs, that many of the proximate systems are exposed to an enhanced flux of ionizing photons, rather than arising in structures of such low density.

One criticism which may be leveled at our photoionization modeling is that it is rather simplistic in its underlying assumptions; we have assumed that $\mathrm{H} \mathrm{I}, \mathrm{C}$ IV, and $\mathrm{O}$ vi absorption arises in the same gas at a uniform temperature and that the ion ratios are determined primarily by the balance between photoionization and recombination. In contrast, S02 found that the kinematics of strong $\mathrm{O}$ vi absorption lines often differ from those of $\mathrm{C}$ IV lines at nearby velocities, and they drew the conclusion that much of the $\mathrm{O}$ VI absorption arises in collisionally ionized gas at a significantly higher temperature than expected from photoionization alone. When we examine the proximate systems studied here, however, we find that in most cases where C IV is detected the velocity match with $\mathrm{O}$ vi is very good, consistent with our assumption of photoionization in the same parcels of gas. One exception was noted above in system A (see Fig. 4), but in the other systems the kinematics of the two ions are mutually consistent.

Another simplistic feature of our modeling has been the assumption that the spectral shape of the ionizing radiation can be 


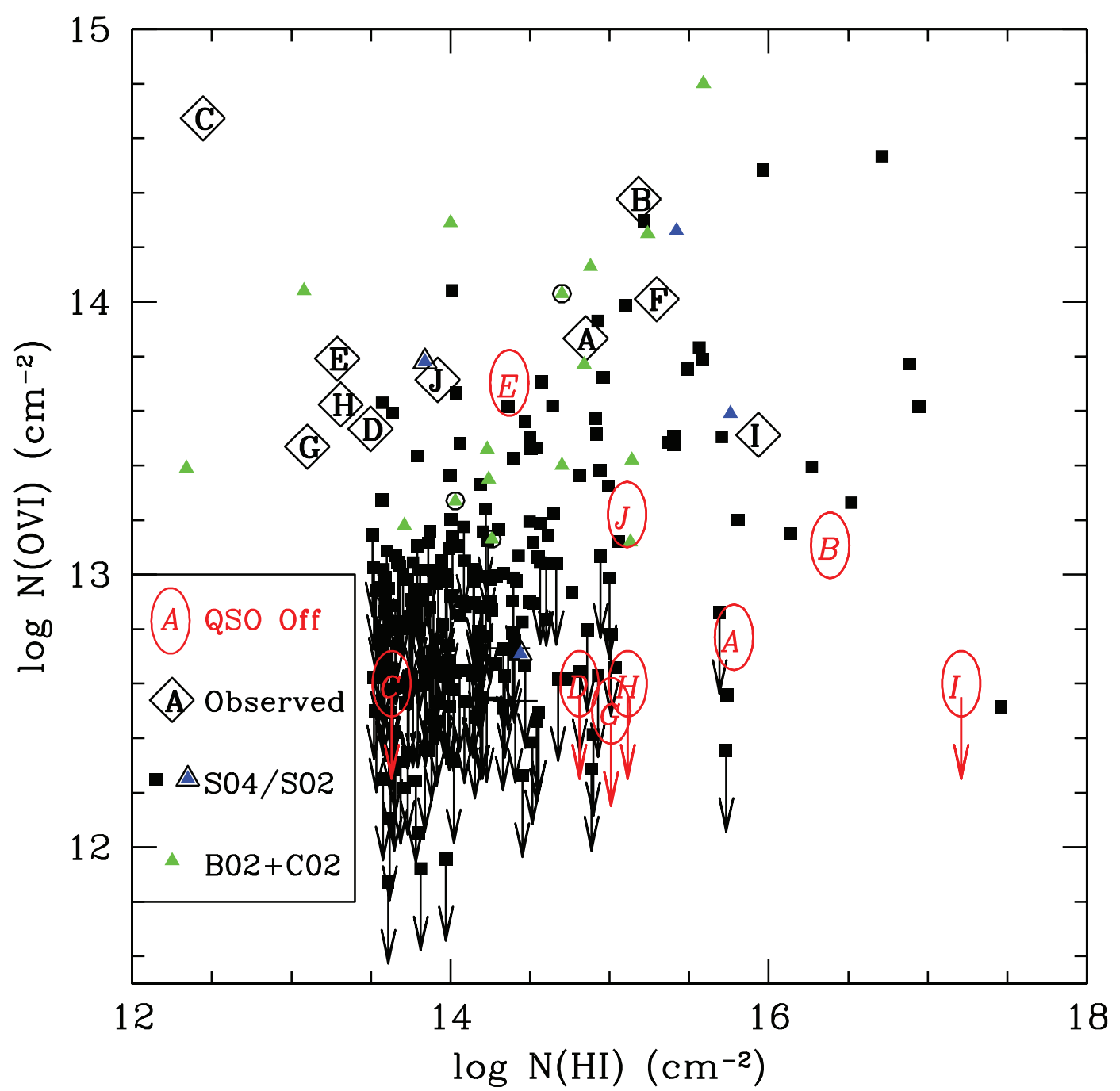

FIG. 14.- Same as Fig. 13, but for O vi. Red ellipses with arrows indicate the approximate detection upper limit for O vi in spectra similar to those used in this paper and

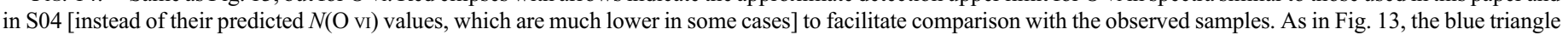
nearest to (observed) system $\mathrm{J}$ indicates a known proximate system near Q1700+64 with $g \simeq 120$.

adequately represented by a power law with spectral index $\alpha=$ $1.8\left[J_{\mathrm{bg}}(\nu) \propto \nu^{-\alpha} ;\right.$ see $\left.\S 4\right]$ and that the nearby QSOs change only the intensity and not the shape of the radiation field to which the gas in each absorption system is exposed. If the UV radiation field from a single QSO dominates over the metagalactic background, then variations in $\alpha$ with time, and between KP 76 and KP 77, might result in different systems seeing radiation fields of different shapes. Such variations may be the reason for the extreme ion ratios measured in system $\mathrm{C}$, which would imply a supersolar metallicity with the ionization spectrum we have assumed (see Fig. 12). For a given $(\mathrm{O} / \mathrm{H})$ ratio, the ratio $N(\mathrm{O} \mathrm{vI}) / N(\mathrm{H} \mathrm{I})$ depends strongly on the spectral shape of the radiation field, as can be appreciated by comparing Figures 12 and 15. Changing the spectral slope $\alpha$ from 1.8 to 2.2 has the effect of lowering the metallicities deduced from photoionization modeling by factors of $\sim 2-3$, because the fraction of oxygen which is 5 times ionized increases. On the other hand, this also has the effect of increasing the ionization parameter $U$ required to reproduce the observed ion ratios, thus implying lower values of $n_{\mathrm{H}}$ for a given boost factor $g$.

We have also investigated the effects on the ion ratios of adopting the more realistic representation of the metagalactic background by F. Haardt \& P. Madau (2005, private communication). The radiation field calculated by these authors broadly resembles the $\alpha=1.8$ power law over the wavelength range of interest, but it also incorporates discrete spectral features resulting from radiative transfer effects in the IGM. With the Haardt \& Madau radiation field, we found that $N(\mathrm{HI})$ remains approximately constant for a given value of $J_{\mathrm{bg}}(\nu)$, but that at a given metallicity $\log N(\mathrm{C}$ IV) and $\log N(\mathrm{O}$ VI $)$ are reduced by $\sim 0.2$ and $\sim 0.6$, respectively. Thus, if the proximate systems considered here were ionized by the metagalactic background rather than the nearby QSOs, their high values of $N(\mathrm{O} \mathrm{vI}) / N(\mathrm{H} \mathrm{I})$ and $N(\mathrm{O} \mathrm{vI}) / N(\mathrm{C}$ IV $)$ would imply even more unusual physical conditions (in terms of $n_{\mathrm{H}}$ and $\rho / \rho_{0}$ ) than deduced above under the power-law assumption.

Finally, we briefly discuss the 13 absorbers without associated metal lines (systems $a-m$ in Tables 2 and 3 ). The dotted and dashdotted lines in Figures 12 and 15 show the loci corresponding to our detection limits $\log N(\mathrm{O}$ vI $) \leq 12.7$ and $\log N(\mathrm{C}$ IV $) \leq 12.1$ for two values of the hydrogen column density that bracket those measured in systems $a-m, \log N(\mathrm{H} \mathrm{I})=13.5$ (dash-dotted line) and 14.5 (dotted line). The most straightforward interpretation of the nondetections of $\mathrm{O}$ VI and $\mathrm{C}$ IV is that these are low-metallicity systems, with $[\mathrm{O} / \mathrm{H}] \lesssim-2$. On the other hand, without the diagnostics provided by the $\mathrm{O}$ and $\mathrm{C}$ ion ratios, we cannot discern the effects of an enhanced radiation field on these systems; it can be readily appreciated from Figures 13 and 14 that the $\mathrm{H}$ I-only systems are also entirely compatible with the values of $N(\mathrm{C}$ IV $) / N(\mathrm{H} \mathrm{I})$ and $N(\mathrm{O} \mathrm{vI}) / N\left(\mathrm{H}_{\mathrm{I}}\right)$ commonly encountered in nonproximate 


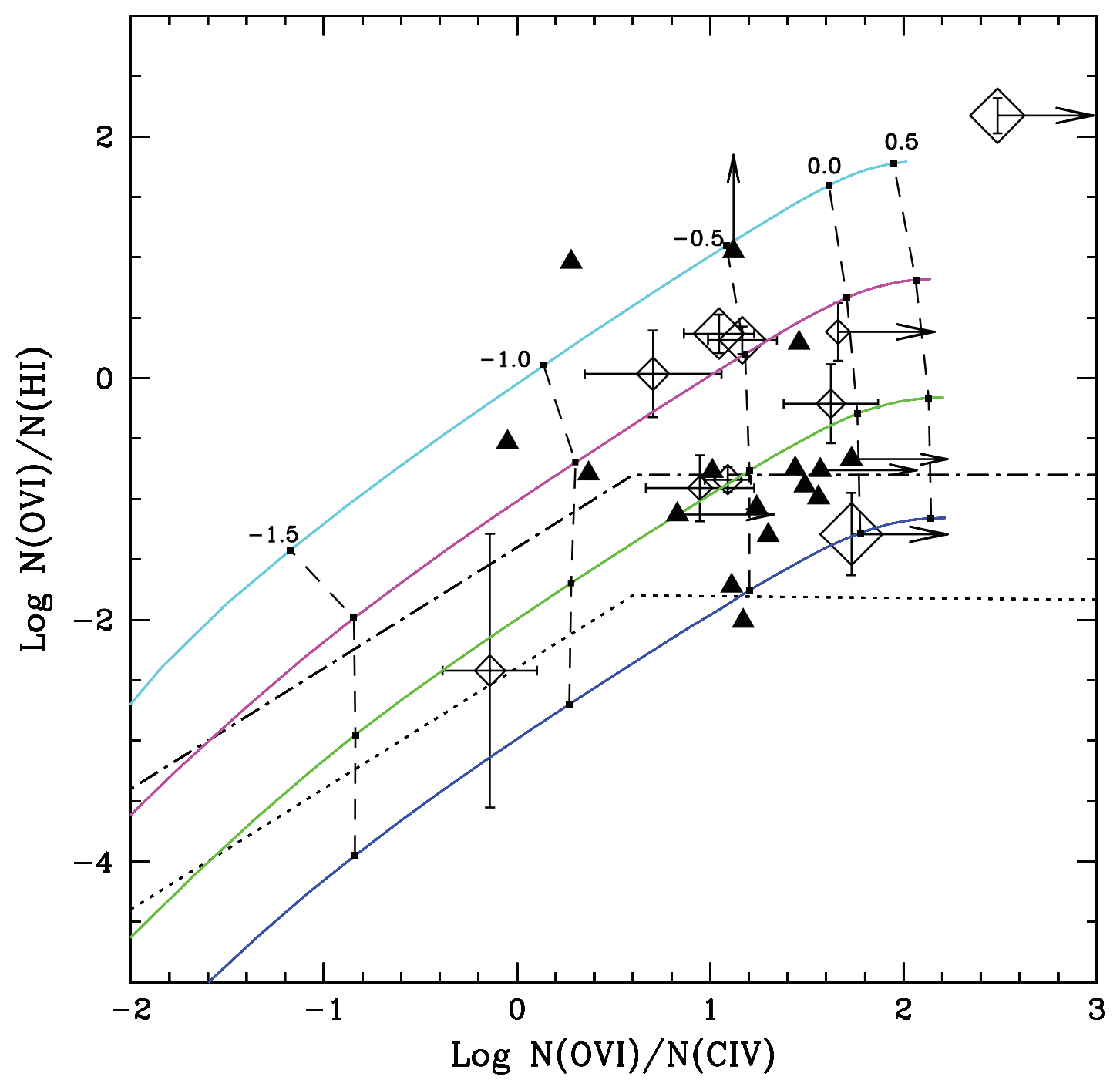

Fig. 15.- Predictions of the CLOUDY photoionization models as in Fig. 12, but using a power-law exponent $\alpha=2.2$ for the incident radiation field. In this case, the measured line ratios would imply lower metallicities and higher ionization parameters by factors of $\sim 2-3$.

systems with the same values of $N\left(\mathrm{H}_{\mathrm{I}}\right)$ as we measure in the proximity regions. In the S04 sample, $62 \%$ of the systems with the same range in $N(\mathrm{H} \mathrm{I})$ are not detected in $\mathrm{O}$ vi to similar limits.

\section{THE LARGE-SCALE ENVIRONMENT OF THE QSOs AND ITS IMPACT ON THE PROXIMITY EFFECT}

\subsection{Galaxies and AGNs in the Q1623+268 Field}

One of the complications in the interpretation of the proximity effect, which has been appreciated since the effect was first recognized, is the likelihood that QSOs are preferentially found in overdensities in the matter distribution. Any such density enhancement relative to a more typical location in the IGM could easily affect the statistics of $\mathrm{H}$ I absorption on the physical scales which are relevant to the proximity effect, whether transverse or along the line of sight. In the present study, we have the advantage of knowing the large-scale distribution of galaxies in the proximity regions of KP 76 and KP 77 from the spectroscopic redshift survey that we have been conducting in the Q1623 +268 field; a full description of the survey and its methods can be found in Steidel et al. (2004) and Adelberger et al. (2005). To date we have cataloged $\sim 300$ objects - star-forming galaxies and AGNs - brighter than $\mathcal{R}=25.5$ at redshifts $z=1.6-3.3$ over an area of sky $\sim 11^{\prime} \times 15^{\prime}$ approximately centered on the KP $76, \mathrm{KP} 77$, and
KP 78 triplet. The redshift distribution of the galaxies and AGNs is shown in Figure 16. The survey is far from complete; in particular, in choosing objects for spectroscopic follow-up we have given preference to color-selected candidates at smaller projected distances from the QSOs on the plane of the sky. Nevertheless, from the observed redshift distribution we can estimate the overdensity of galaxies and fainter AGNs (in redshift space) compared to a sample drawn randomly from the overall redshift selection function of the survey (also shown in Fig. 16).

Within the field where spectroscopic follow-up has been carried out, there are 19,19, and 20 objects with redshifts which place them within $\pm 1500 \mathrm{~km} \mathrm{~s}^{-1}$ of the systemic redshifts of KP 76, KP 77, and KP 78, respectively. ${ }^{7}$ For comparison, the

\footnotetext{
${ }^{7}$ Among these objects, three are faint QSOs $(\mathcal{R}=19.38,22.70$, and 23.95) at redshifts near that of KP 76, and one is an $\mathcal{R}=20.44$ QSO at a redshift near that of KP 77. This last one, Q1623-BX 603, is located $97^{\prime \prime}(780 \mathrm{kpc})$ from the sight line to KP 78. With a redshift $z_{\mathrm{em}} \simeq 2.530$, estimated from its rest-frame UV emission lines, its peak radiation would be expected to be near $-425 \mathrm{~km} \mathrm{~s}^{-1}$ (Fig. 3 , middle) with a boost factor $g \simeq 7$ relative to the background. If its redshift is correct (we have not obtained NIRSPEC spectroscopy of this QSO), its contribution to the local radiation field at $-425 \mathrm{~km} \mathrm{~s}^{-1}$ is $\sim 10 \%$ of that of KP 77 . None of the other QSOs make a significant contribution to the radiation field in the proximity regions considered.
} 


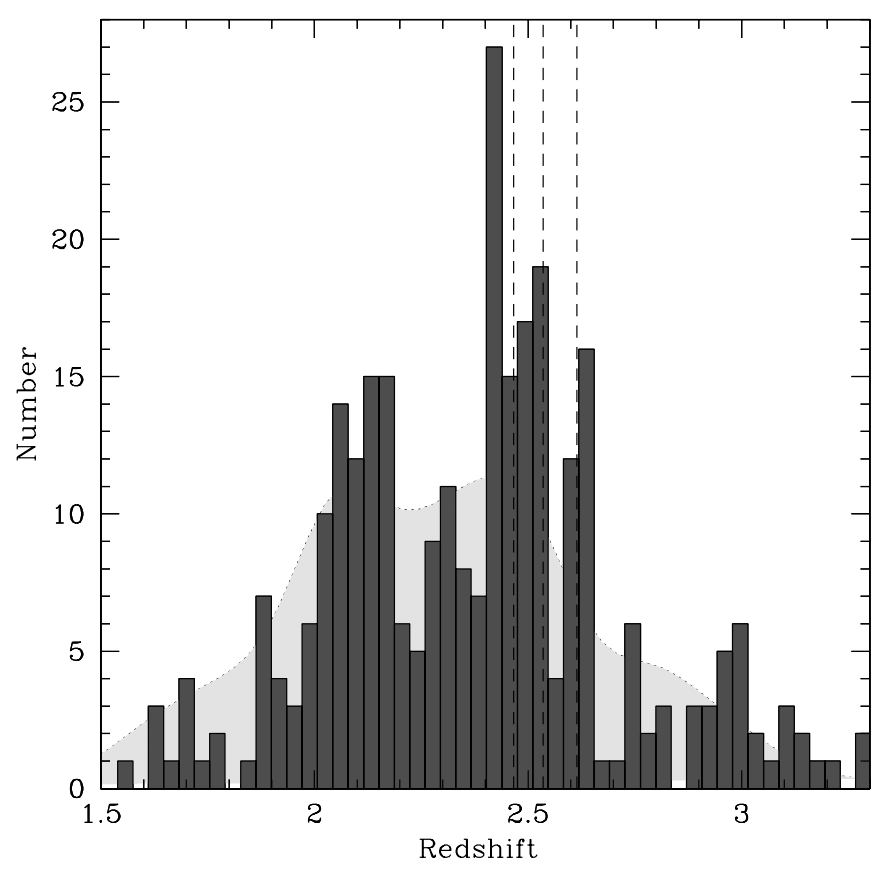

FIG. 16.- Redshift distribution of 298 spectroscopically confirmed star-forming galaxies and AGNs in the Q1623+268 field, selected by their rest-frame UV colors. Galaxies have been grouped in redshift bins of width $\Delta v= \pm 1500 \mathrm{~km} \mathrm{~s}^{-1}$ so as to match the QSO proximity regions we have been considering. The redshifts of KP 76, KP 77, and KP 78 are indicated with vertical dashed lines. The light, smooth curve in the background shows the overall redshift selection function of a random sample of 298 objects selected using the same color selection criteria (in the absence of clustering), based on the full spectroscopic sample of $\sim 2000$ galaxies in our ongoing survey. The three QSOs are located within moderate overdensities in the underlying distribution of star-forming galaxies. [See the electronic edition of the Journal for a color version of this figure.]

corresponding number of objects expected in the same redshift intervals for an unclustered population are 10, 9, and 6, respectively. Thus, the three QSOs appear to reside in moderate galaxy overdensities: $\delta \rho / \rho \simeq 1$ for KP 76 and KP 77, while KP 78 lies in the most significant redshift space overdensity with $\delta \rho / \rho \simeq 2$. However, these are the overdensities relative to the population of star-forming galaxies at $z \sim 2.5$ (the BX galaxies of Steidel et al. 2004), which themselves are significantly biased relative to the underlying mass distribution at these redshifts; Adelberger et al. (2005) measured a comoving correlation length $r_{0} \simeq 4 \mathrm{Mpc}$, which corresponds roughly to a linear bias factor of $b \simeq 2$. Thus, even regions containing an average density of BX galaxies would probably represent overdensities in the matter distribution. Of course, what we are interested in is the overdensity in $\mathrm{H}$ I compared to an average location in the universe at these redshifts. While it is uncertain how to relate the overdensity of BX galaxies to that of $\mathrm{H}$ I [the factor relating the two presumably depends on the threshold $N(\mathrm{H} \mathrm{I})$ ], it seems reasonable to conclude from the above that the Ly $\alpha$ forest near KP 76 and KP 77 is denser by a factor of a few compared to an average location in the IGM.

KP 76, KP 77, and KP 78 are not unusual in being located in galaxy overdensities; rather, their environments are consistent with the galaxy-AGN cross-correlation function measured from a much larger sample by Adelberger \& Steidel (2005). It is therefore not surprising that the small samples of QSO pairs used so far to search for the TPE have found little evidence to support it based on the expected decrease in H I optical depth; it is easy to see how the overdensity of relatively high $N(\mathrm{H}$ I $)$ systems in the QSO environments can more than compensate for the loss of ab- sorption from optically thin systems whose Ly $\alpha$ line equivalent width would be most affected by an enhanced UV radiation field.

Strong correlations between galaxies and high $N(\mathrm{H}$ I) absorption systems are known to exist on scales of a few hundred (proper) kiloparsecs at $z \simeq 2.5$ (Adelberger et al. 2003, 2005; Simcoe et al. 2006). Figure 17 shows the locations of the three QSOs together with the objects from the spectroscopic sample that lie within the proximity regions $\left( \pm 1500 \mathrm{~km} \mathrm{~s}^{-1}\right)$ of the foreground QSOs KP 76 and KP 77. Note that there are three galaxies within the proximity region of each of KP 76 and KP 77 located within $60^{\prime \prime}$ $(\sim 0.5$ proper $\mathrm{Mpc}$ in the transverse direction at $z=2.5)$ of the line of sight to KP 78. Similarly, there is one galaxy within the proximity region of KP 76 located less than $0.5 \mathrm{Mpc}$ from the sight line to KP 77. The redshifts of the galaxies and their distances from the QSO sight lines are listed in Table 5; the velocities of the galaxies relative to the foreground QSOs are also indicated with yellow shading in Figure 3. The galaxy redshifts were determined from their rest-frame UV interstellar absorption lines and/or Ly $\alpha$ emission after correcting for the systematic velocity offsets of these spectral features from the systemic redshift; the error in this procedure is $\pm 140 \mathrm{~km} \mathrm{~s}^{-1}$ (C. C. Steidel et al., in preparation). For two of the galaxies, BX 522 and MD 107, we were able to measure the systemic redshift directly from NIRSPEC observations of their $\mathrm{H} \alpha$ emission lines with a reduced error of $\pm 60 \mathrm{~km} \mathrm{~s}^{-1}$ (Erb et al. 2006).

Referring to Figures 3 and 17, it is interesting to try to associate galaxies and absorption systems within the proximity regions of KP 76 and KP 77. The galaxy within a proximity region which lies closest to the sight line to a background QSO is MD 126 (within the proximity region of KP 76 and only $133 \mathrm{kpc}$ from the sight line to KP 78). Its redshift matches that of system B very well (see Fig. 3). As a matter of fact, systems A and B exhibit similar ionization parameters and metallicities (from our photoionization modeling in Fig. 12), so they could both be due to MD 126 (in which case their velocity difference would be due to peculiar motions rather than the Hubble flow, as was assumed in calculating their distances from the sight line to KP 78). The redshift of the next closest galaxy, BX 522, $175 \mathrm{kpc}$ from the KP 78 sight line and also in the KP 76 proximity region, is intermediate between those of systems $\mathrm{D}$ and $\mathrm{E}$, which are separated by $\Delta v \simeq$ $300 \mathrm{~km} \mathrm{~s}^{-1}$ and have metallicities $[\mathrm{O} / \mathrm{H}] \gtrsim-1$. The third galaxy within the proximity region of $\mathrm{KP} 76, \mathrm{BX} 546$, is within $120 \mathrm{~km} \mathrm{~s}^{-1}$ of system $\mathrm{C}$, at a transverse distance of $438 \mathrm{kpc}$. Turning to galaxies close to the sight line to KP 78 but in the proximity region of KP 77, MD 106 is within $130 \mathrm{~km} \mathrm{~s}^{-1}$ of systems $b$ and $c$, while MD 107 is within $100 \mathrm{~km} \mathrm{~s}^{-1}$ of system F (which in our modeling would have the properties of a DLA in the absence of the radiation field from KP 77). Near the sight line to KP 77, galaxy BX 435 is within $40 \mathrm{~km} \mathrm{~s}^{-1}$ of system $m$, and within the uncertainties it has the same redshift as systems $l$ and $m$. None of these possible associations between galaxies and absorption lines are particularly unusual or unexpected, compared to regions lacking bright QSOs but having similar galaxy overdensities (Adelberger et al. 2003, 2005). Similarly, given the level of incompleteness of the spectroscopic sample, the lack of identified galaxies associated with other absorption systems in the proximity regions should not be taken to imply that such galaxies are not present.

\subsection{Matter Overdensities and the Proximity Effect}

Faucher-Giguere et al. (2008) have recently presented a theoretical investigation of how the environments which are likely to 


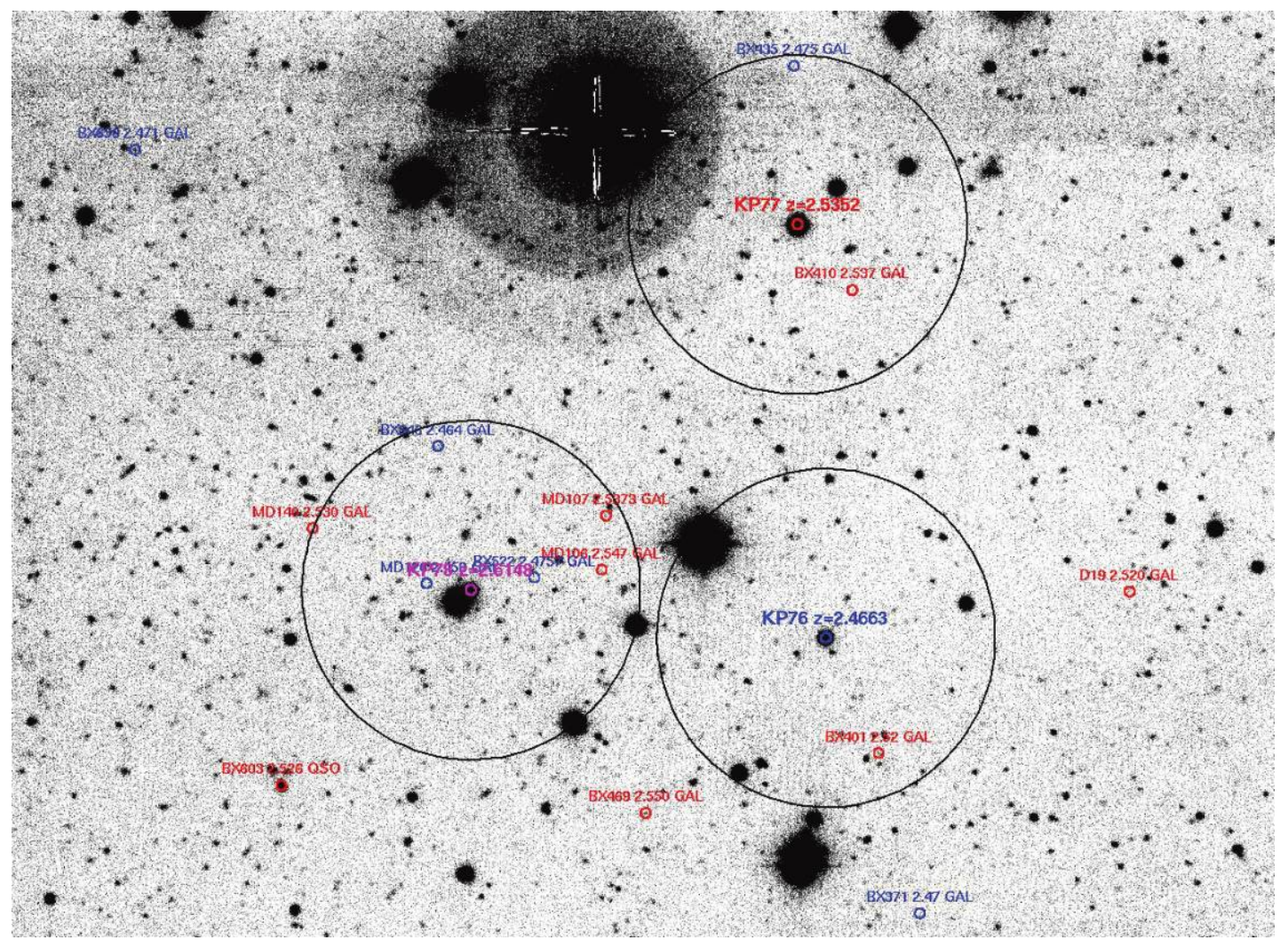

FIG. 17. - The $u^{\prime}$-band image of the Q1623 +268 field showing the locations of the three bright QSOs and spectroscopically confirmed galaxies within the $\pm 1500 \mathrm{~km} \mathrm{~s}{ }^{-1}$ proximity regions of the two foreground QSOs. The black circles are $60^{\prime \prime}$ in radius (corresponding to 0.48 proper Mpc in the transverse direction at $z=2.5$ ). Galaxies and faint QSOs in the proximity region of KP 76 are shown in blue, while those in the proximity region of KP 77 are labeled in red. North is up and east to the left.

host QSOs may bias the inferred value of the hydrogen photoionization rate from the metagalactic background, $\Gamma_{\mathrm{bg}}$, at redshifts $z=2-4$. These authors concluded that overdense environments would lead to overestimates of $\Gamma_{\text {bg }}$ by a factor of $\sim 2.5$ in determinations of the proximity effect based on the distribution of Ly $\alpha$ optical depths $\tau_{\mathrm{H}_{\mathrm{I}}}$. The bias arises from a combination of larger IGM density (resulting in larger values of $\tau_{\mathrm{H}_{\mathrm{I}}}$ ) and the infall of surrounding material onto the massive halos in which the QSOs are likely to reside. Such effects would lead one to conclude that the boost by the QSO radiation to the ionizing flux seen by nearby clouds is smaller than it really is, thereby leading to an overestimate of the metagalactic background.

TABLE 5

Spectroscopically Identified Galaxies in $f_{Q}$ Proximity Regions

\begin{tabular}{|c|c|c|c|c|c|c|}
\hline Galaxy & BQSO & FQSO & $\Delta \theta^{\mathrm{a}}$ & $\begin{array}{c}d^{\mathrm{b}} \\
(\mathrm{kpc})\end{array}$ & $z_{\text {gal }}$ & $\Delta v_{\mathrm{gal}}^{\mathrm{c}}$ \\
\hline MD $126 \ldots \ldots \ldots . .$. & KP 78 & KP 76 & 16.6 & 133 & 2.458 & $-720 \pm 180$ \\
\hline BX 522.............. & KP 78 & KP 76 & 21.9 & 175 & 2.4757 & $+810 \pm 90$ \\
\hline BX 546.............. & KP 78 & KP 76 & 54.1 & 433 & 2.465 & $+200 \pm 180$ \\
\hline MD $106 \ldots \ldots \ldots . .$. & KP 78 & KP 77 & 46.2 & 370 & 2.547 & $-1000 \pm 180$ \\
\hline MD $107 \ldots \ldots \ldots . .$. & KP 78 & KP 77 & 54.1 & 433 & 2.5373 & $+180 \pm 90$ \\
\hline MD $140 \ldots \ldots \ldots . .$. & KP 78 & KP 77 & 60.2 & 482 & 2.530 & $-440 \pm 180$ \\
\hline BX 435............... & KP 77 & KP 76 & 56.0 & 448 & 2.474 & $+750 \pm 180$ \\
\hline
\end{tabular}

Note.-Galaxies within $60^{\prime \prime}$ ( $\sim 0.5 \mathrm{Mpc}$ ) of background QSO (BQSO) sight lines and within the proximity region of the foreground QSOs (FQSO); see Fig. 17.

${ }^{a}$ Angular separation (in arcseconds) between galaxy and BQSO sight lines.

${ }^{\mathrm{b}}$ Projected physical distance between BQSO sight line and galaxy, at $z_{\mathrm{gal}}$.

${ }^{c}$ Velocity difference $\left(\mathrm{km} \mathrm{s}^{-1}\right)$ between galaxy systemic redshift and that of the foreground QSO (cf. Fig. 3). The quoted errors include estimates of the uncertainties of both the galaxy and the QSO systemic redshifts.
An additional complication is that essentially all previous measures of the proximity effect have been based either on counting the number of Ly $\alpha$ lines above a given equivalent width limit or calculating the total transmitted flux near the redshift of the QSO, and then comparing this statistic with expectations for the general IGM at the same redshift. This approach has been dictated by the fact that the resolution of most spectra used to search for the proximity effect is too coarse to determine $\tau_{\mathrm{H}_{\mathrm{I}}}$ directly. The problem is that the response of the line equivalent width to changes in $\tau_{\mathrm{H}_{\mathrm{I}}}$ is nonlinear. The lines which are most sensitive to changes in $\tau_{\mathrm{H}_{\mathrm{I}}}$ are those with $\tau_{\mathrm{H}_{\mathrm{I}}}<1$ (on the linear part of the curve of growth), with equivalent widths which are generally too small to be included in the samples used to measure the proximity effect. On the other hand, the Ly $\alpha$ lines which are included typically have column densities in the range $14 \lesssim \log N(\mathrm{H} \mathrm{I}) \lesssim 17$, where the equivalent width is a highly insensitive measure of $\tau_{\mathrm{H}_{\mathrm{I}}}$ (such lines fall on the flat part of the curve of growth). Consequently, the sensitivity of Ly $\alpha$ line counts to a boost in the ionizing radiation field within the proximity regions depends sensitively on line equivalent width. In spectra of moderate resolution and $\mathrm{S} / \mathrm{N}$, a situation may arise whereby the detection limit for the $\operatorname{Ly} \alpha$ line equivalent width is sufficiently high that the increase in strong Ly $\alpha$ lines due to the local matter overdensity can mask an overall reduction in $\tau_{\mathrm{H}_{\mathrm{I}}}$ due to the enhanced ionizing flux, except in very small regions very close to the QSO. In cases where the QSO redshift has been underestimated by $1000-2000 \mathrm{~km} \mathrm{~s}^{-1}$ from restframe UV emission lines, the region most affected by the QSO radiation field may not even be considered in typical proximity effect measurements!

In summary, the number per unit redshift of the relatively strong Ly $\alpha$ lines that have been used in most previous measurements of the QSO proximity effect is likely to be closely related to the matter 
overdensity in the surrounding volumes. While this gas will, of course, be affected by the QSO radiation field, the presence of the QSO could easily be secondary to the local environment in dictating the statistics of such lines. Once a more secure relationship is established between local galaxy density and the incidence of relatively high $N(\mathrm{HI})$ systems, it may be possible to use the observed galaxy density to calibrate out the environmental dependence on a QSO-by-QSO basis. On the other hand, because QSOs tend to inhabit relatively dense environments, one is more likely to benefit from the presence of systems containing lines of highly ionized metals which, as shown here, may offer the strongest observational constraints on the nature of the local radiation field.

\section{SUMMARY AND DISCUSSION}

Using high-resolution spectra of a triplet of QSOs with transverse separations of $\sim 1 \mathrm{Mpc}$ at $z \simeq 2.5$, together with accurate determinations of the QSO systemic redshifts from rest-frame optical emission lines and extensive spectroscopic observations of galaxies and AGNs in the same field, we have conducted the most detailed investigation to date of the transverse proximity effect (TPE). By focusing on the regions of the IGM where the QSOs should overwhelm the metagalactic radiation field by factors between $\sim 10$ and $\sim 200$ - if they have radiated isotropically and with similar luminosities over the past $0.2-30 \mathrm{Myr}$-we have examined the details of the ionization state of individual metalabsorption systems, rather than counting Ly $\alpha$ lines above a given equivalent width threshold, as has generally been done in previous attempts to measure the TPE. We have shown that the 10 metalline systems within the proximity regions of the two foreground QSOs have properties that are more easily explained if they are being illuminated by a UV radiation field significantly more intense than the metagalactic background. Using photoionization models, we have shown that most of the observed absorption systems have properties consistent with normal (i.e., nonproximate) intermediate column density $[\log N(\mathrm{H}$ I $) \sim 14.5-16.5]$ metalline systems that have been ionized by QSO continuum radiation with intensity consistent with that inferred from the observed fluxes of the two foreground QSOs.

We have placed the observed QSOs in the context of the largescale distribution of galaxies in the same field, showing that all three lie in regions of moderate galaxy overdensity, conditions typical of high-redshift QSOs and AGNs. Even with accurate QSO redshifts (which correct the published values for the same QSOs by $1000-2000 \mathrm{~km} \mathrm{~s}^{-1}$ ), the naive expectation that there should be a dearth of Ly $\alpha$ absorption systems in the proximity regions of the foreground QSOs is not supported by the data. We argue that the environments of the QSOs and an enhancement of moderate column density $\mathrm{H}$ I absorption compared to average locations in the IGM can easily mask the effects of the TPE if one relies on counting statistics rather than examining the details of the gas-phase physical conditions.

\subsection{QSO Lifetimes and Isotropy}

In principle, it should be possible to use information on the distribution of the gas that is clearly affected by QSO radiation relative to the positions and redshifts of the foreground QSOs to measure or set limits on both the lifetime and the isotropy of the QSO radiation field. Each absorption system listed in Table 3 and indicated in Figure 3 samples a different time interval $\Delta t$ and boost factor $g$ under the simple hypothesis that the QSOs shine isotropically and at constant luminosity over their radiative lifetimes. By comparing our sample to "proximate" and "nonproximate" absorption systems from the literature, as in Figures 13 and 14, and from arguments based on cloud density and size, we have shown that most of the systems in our sample-systems C, D, E, F, G, $\mathrm{H}$, and $\mathrm{J}$ - are probably being overionized by the nearby QSO, while systems A and B are merely consistent with that hypothesis. Taken at face value, the spread of values of $\Delta t$ in Table 3 then implies that the minimum QSO lifetime is $\sim 25 \mathrm{Myr}$ for KP 76 (from system E) and $\sim 16$ Myr for KP 77 (from system H). As discussed above, system I, in the proximity region for KP 77, is the only system whose properties may favor ionization by the metagalactic field alone, without local enhancement; again, taken at face value, this would indicate a radiative lifetime for KP 77 in the range $16 \mathrm{Myr}<\Delta t<33 \mathrm{Myr}$. Radiative lifetimes of $20-$ $30 \mathrm{Myr}$ are entirely consistent with He II TPE measurements by Jakobsen et al. (2003) and with numerous estimates based on QSO duty cycle arguments (e.g., Steidel et al. 2002) or the local $M_{\mathrm{bh}}-\sigma$ relation between supermassive black holes and their host galaxies (e.g., Martini \& Weinberg 2001).

In reality, there are many other variables which potentially could modify such conclusions, even accepting the evidence that at least some of the absorption systems are overionized due to their proximity to a bright QSO. For example, even if the lifetime of a QSO event is a well-defined quantity, it is unlikely that an accreting supermassive black hole maintains a constant luminosity throughout its active phase; rather, its accretion rate and UV output could vary intermittently or grow (or decay) exponentially. Because each absorption system samples a different time in the QSO's history, it is entirely possible that systems whose distances from the QSO are larger (so that the $g$-factor is smaller) could have experienced a more intense radiation field in the past, or that a system at some intermediate value of $\Delta t$ happened to coincide with a dormant period in the QSO's radiative history. We have also seen that the effects of an enhanced radiation field can be quite subtle. It is not always possible to say with confidence, even with highquality data, whether or not a given absorber is experiencing an enhanced ionizing radiation field, because we have no knowledge of its environment or physical conditions prior to the time the QSO began radiating at its present luminosity.

Given all these caveats, the strongest statement we can make about the isotropy of the QSO radiation is that there is no evidence for anisotropy in the present data. If the QSOs' radiation were significantly beamed, one might reasonably expect to find absorption systems well within the proximity zones with properties that are inconsistent with the assumption of a radiation field intensity significantly boosted over the metagalactic background. Within the two proximity regions considered, the only absorption system that seems marginally inconsistent with the assumption of an enhanced radiation field (system I) also has the largest value of $\Delta t$ and a relatively small boost factor $g$.

As part of our survey of the Q1623+268 field, we obtained deep Spitzer IRAC and MIPS images which include the QSO triplet. We found that KP 76, KP 77, and KP 78 have nearly identical spectral energy distributions between 0.35 and $24 \mu \mathrm{m}$, with flat spectra $\left(f_{\nu} \simeq\right.$ constant) between 0.35 and $4.5 \mu \mathrm{m}$ and with $\nu f_{\nu}(0.36 \mu \mathrm{m}) / \nu f_{\nu}(24 \mu \mathrm{m}) \simeq 4$. QSOs that are heavily obscured over a large solid angle would be expected to be very bright in the thermal IR due to emission from heated dust. While modeling of the QSO spectral energy distributions is beyond the scope of this paper, the data suggest that the two foreground QSOs (as well as $\mathrm{KP} 78$, although it does not matter for the present purposes) are not heavily obscured over a large fraction of $4 \pi \mathrm{sr}$, given the relatively weak $24 \mu \mathrm{m}$ luminosity. This provides independent support for the hypothesis that these three QSOs would be seen as UV-bright over a large fraction of a $4 \pi$ sr solid angle. 
The results we have reported provide a counterexample to recent claims of an excess of high $N(\mathrm{H}$ I $)$ absorption systems near the redshifts of foreground QSOs in the spectra of background QSOs compared to line-of-sight proximate absorbers with the same characteristics (e.g., Bowen et al. 2006; Hennawi et al. 2006). These authors interpret such an excess as evidence for anisotropy of the QSO radiation which presumably (over)ionizes the clouds in the line of sight to Earth but is not seen (with the same intensity) by gas at transverse distances. One difference that must be borne in mind is that the analysis by Hennawi et al. (2006) refers to absorption systems with column densities $\log N(\mathrm{H} \mathrm{I})>$ 19 , more than 3.5 orders of magnitude higher than any in the sample considered here. Similarly, the work by Bowen et al. (2006) targeted strong $\mathrm{Mg}$ II absorbers which are likely to be Lyman limit systems with $\log N(\mathrm{H} \mathrm{I})>17.5$. While our data appear to be inconsistent with significant beaming of the ionizing radiation from KP 76 and KP 77, it is certainly possible that QSOs may differ in the solid angle over which they radiate, and that the apparent differences between our study and those referenced above is due to such variations from QSO to QSO. Finally, while we cannot rule out the possibility that there may be an anisotropic distribution of gas (as opposed to anisotropic ionization) surrounding many QSOs, it would be hard to understand if such anisotropy extended over physical scales of $\sim 1 \mathrm{Mpc}$, as would be required to explain some of the observations.

A lingering concern is the uncertainty in the relevant distances introduced by the systematic errors in the QSO systemic redshifts. For example, it is debatable whether it is surprising or not to find high $N(\mathrm{H} \mathrm{I})$ absorbers near the redshift of a foreground QSO. Even in cases where the projected sight lines pass within tens of kiloparsecs of one another, if the uncertainty in the redshift of the foreground QSO is $\sim 1500 \mathrm{~km} \mathrm{~s}^{-1}$ (equivalent to a distance of $\pm 6 \mathrm{Mpc}$ at $z=2.5$ ), the relevant $g$-factor could be uncertain by up to 3 orders of magnitude. Many such ambiguities could be addressed with more accurate redshifts for the QSOs, as well as more accurate values of $N(\mathrm{H} \mathrm{I})$ and other indicators of the physical conditions in the gas.

\subsection{The Elusive Transverse Proximity Effect}

In this paper we have used a particularly well-observed triplet of QSOs in a concerted effort to test for the presence of the TPE. We have shown that in spite of the absence of an obvious "clearing" in the Ly $\alpha$ forest near the redshifts of bright foreground QSOs, significant evidence for local enhancements in the ionizing radiation field is present when one examines the detailed physical conditions of metal-line systems within $5 \mathrm{Mpc}$ of the foreground QSOs. Crucial to this analysis are (1) the ability to measure accurate $\mathrm{H}$ i column densities (for which echelle spectra extending to at least $\mathrm{Ly} \beta$ are required); (2) knowing as precisely as possible where to expect the influence of the foreground QSOs (for which the forbidden-line spectroscopy of the QSOs was essential); (3) the ability to detect weak lines of highly ionized metallic species, in this case $\mathrm{C}$ IV and $\mathrm{O}$ VI; and (4) knowledge of the large-scale environment inhabited by the foreground QSOs, since galaxy overdensities are likely to be accompanied by the presence of appreciable $\mathrm{H}$ i absorption. ${ }^{8}$

Essentially, all previous searches for the TPE have been missing most or all of these ingredients. As discussed above, reliance on relatively crude line counting or mean flux measurements in the forest; the likelihood that the redshifts of the foreground QSOs, even when taken from carefully compiled catalogs such as SDSS, are incorrect by as much as $2000 \mathrm{~km} \mathrm{~s}^{-1}$ (an error $\delta z>$ $1000 \mathrm{~km} \mathrm{~s}^{-1}$ seems quite typical); and density enhancements of galaxies and intergalactic gas local to the foreground QSOs could conspire to mask the TPE. In view of our results, these effects seem at least as plausible as anisotropic emission, short-timescale variability, or very short QSO lifetimes in explaining the difficulties experienced so far in detecting the TPE. Based on the case investigated in this paper, the null hypothesis that bright QSOs radiate isotropically over characteristic timescales of a few $10^{7} \mathrm{yr}$ (timescales suggested by many less-direct arguments) is consistent with the observations. Whether this is the rule rather than the exception could be established using similar observations of other fields with multiple QSO sight lines. Somewhat farther in the future, the technique can be improved and extended using background galaxies which would provide much finer spatial and temporal sampling of the response of the IGM to radiation from QSOs (Adelberger 2004).

We would like to thank Dawn Erb, Naveen Reddy, and Alice Shapley for their collaboration in the large survey which supplied the LRIS spectroscopic redshifts of galaxies and AGNs in the field of Q1623+268. In addition, Dawn and Alice obtained the near-IR spectra used to determine the systemic redshifts of the QSOs, and Naveen kindly provided the Spitzer results on the QSOs prior to publication. Kurt Adelberger is thanked for many discussions and for his participation in the early stages of the project. We would also like to thank Tom Barlow, Rob Simcoe, George Becker, and Bob Carswell for assistance with the software used to reduce HIRES data. Rob Simcoe also provided us with measurements from his previous work, for which we are grateful. Wal Sargent provided us with his HIRES spectra of KP 76 and KP 77, which were combined with the new data presented here. An anonymous referee and Juna Kollmeier provided constructive comments which significantly improved the paper. Finally, we wish to extend thanks to those of Hawaiian ancestry on whose sacred mountain we are privileged to be guests. This work was supported by grants AST 03-07263 and AST 06-06912 from the US National Science Foundation and by the David and Lucile Packard Foundation.

\footnotetext{
8 Alternatively, as shown by Jakobsen et al. (2003) and Worseck \& Wisotzki (2006), using the $\mathrm{He}_{\mathrm{II}}$ transitions in concert with $\mathrm{H}_{\mathrm{I}}$ is a very powerful technique, because the effects of an enhancement in the radiation field by a nearby QSO are then much more evident than if one has access to $\mathrm{H}_{\mathrm{I}}$ lines only. However, the downside of this approach is that, given the short rest-frame wavelengths of the He II Lyman series, there is only a handful of sight lines known at present where this technique can be applied in practice; in most cases optically thick intervening absorbers in the $\mathrm{H}$ I Lyman continuum prevent measurement of the He II lines.
}

\section{REFERENCES}

Adelberger, K. L. 2004, ApJ, 612, 706

Adelberger, K. L., Shapley, A. E., Steidel, C. C., Pettini, M., Erb, D. K., \& Reddy, N. A. 2005, ApJ, 629, 636

Adelberger, K. L., \& Steidel, C. C. 2005, ApJ, 630, 50

Adelberger, K. L., Steidel, C. C., Kollmeier, J. A., \& Reddy, N. A. 2006, ApJ, 637,74

Adelberger, K. L., Steidel, C. C., Shapley, A. E., \& Pettini, M. 2003, ApJ, 584, 45

Bajtlik, S., Duncan, R. C., \& Ostriker, J. P. 1988, ApJ, 327, 570
Bergeron, J., Aracil, B., Petitjean, P., \& Pichon, C. 2002, A\&A, 396, L11 (B02) Bergeron, J., \& Herbert-Fort, S. 2005, in IAU Colloq. 199, Probing Galaxies through Quasar Absorption Lines, ed. P. R. Williams, C. Shu, \& B. Menard (Cambridge: Cambridge Univ. Press), 265

Bolton, J. S., Haehnelt, M. G., Viel, M., \& Springel, V. 2005, MNRAS, 357, 1178

Boroson, T. 2005, AJ, 130, 381

Bowen, D. V., et al. 2006, ApJ, 645, L105 
Cantalupo, S., Porciani, C., Lilly, S. J., \& Miniati, F. 2005, ApJ, 628, 61

Carswell, R. F., Schaye, J., \& Kim, T.-S. 2002, ApJ, 578, 43 (C02)

Croft, R. A. C. 2004, ApJ, 610, 642

Crotts, A. P. S. 1989 , ApJ, 336, 550

Crotts, A. P. S., Burles, S., \& Tytler, D. 1997, ApJ, 489, L7

Crotts, A. P. S., \& Fang, Y. 1998, ApJ, 502, 16

Erb, D. K., Steidel, C. C., Shapley, A. E., Pettini, M., Reddy, N. A., \& Adelberger, K. L. 2006, ApJ, 646, 107

Espey, B. R., Carswell, R. F., Bailey, J. A., Smith, M. G., \& Ward, M. J. 1989, ApJ, 342, 666

Faucher-Giguere, C.-A., Lidz, A., Zaldarriaga, M., \& Hernquist, L. 2008, ApJ, 673,39

Ferland, G. J., Korista, K. T., Verner, D. A., Ferguson, J. W., Kingdon, J. B., \& Verner, E. M. 1998, PASP, 110, 761

Fernández-Soto, A., Barcons, X., Carballo, R., \& Webb, J. K. 1995, MNRAS, 277,235

Francis, P. J., \& Bland-Hawthorn, J. 2004, MNRAS, 353, 301

Gallerani, S., Ferrara, A., Fan, X., \& Choudhury, T. R. 2008, MNRAS, in press (arXiv: 0706.1053)

Gould, A., \& Weinberg, D. H. 1996, ApJ, 468, 462

Haehnelt, M. G., Natarajan, P., \& Rees, M. J. 1998, MNRAS, 300, 817

Hennawi, J. F., et al. 2006, ApJ, 651, 61

Hosokawa, T. 2002, ApJ, 576, 75

Hunt, M. P., Steidel, C. C., Adelberger, K. L., \& Shapley, A. E. 2004, ApJ, 605, 625

Jakobsen, P., Jansen, R. A., Wagner, S., \& Reimers, D. 2003, A\&A, 397, 891
Martini, P. 2004, in Coevolution of Black Holes and Galaxies, ed. L. C. Ho (Cambridge: Cambridge Univ. Press), 169

Martini, P., \& Weinberg, D. H. 2001, ApJ, 547, 12

McLean, I. S., et al. 1998, Proc. SPIE, 3354, 566

Murdoch, H. S., Hunstead, R. W., Pettini, M., \& Blades, J. C. 1986, ApJ, 309, 19

Richstone, D., et al. 1998, Nature, 395, A14

Sargent, W. L. W., Young, P., \& Schneider, D. P. 1982, ApJ, 256, 374

Schirber, M., Miralda-Escudé, J., \& McDonald, P. 2004, ApJ, 610, 105

Scott, J., Bechtold, J., Dobrzycki, A., \& Kulkarni, V. P. 2000, ApJS, 130, 67

Shapley, A. E., Steidel, C. C., Pettini, M., Adelberger, K. L, \& Erb, D. K. 2006, ApJ, 651, 688

Simcoe, R. A., Sargent, W. W., \& Rauch, M. 2002, ApJ, 578, 737 (S02) 2004, ApJ, 606, 92 (S04)

Simcoe, R. A., Sargent, W. W., Rauch, M., \& Becker, G. 2006, ApJ, 637, 648

Sramek, R. A., \& Weedman, D. A. 1978, ApJ, 221, 468

Steidel, C. C., Hunt, M. P., Shapley, A. E., Adelberger, K. L., Pettini, M., Dickinson, M., \& Giavalisco, M. 2002, ApJ, 576, 653

Steidel, C. C., Shapley, A. E., Pettini, M., Adelberger, K. L., Erb, D. K., Reddy, N. A., \& Hunt, M. P. 2004, ApJ, 604, 534

Tytler, D., et al. 2004, ApJ, 617, 1

Vogt, S. S., et al. 1994, Proc. SPIE, 2198, 362

Worseck, G., Fechner, C., Wisotzki, L., \& Dall'Aglio, A. 2007, A\&A, 473, 805

Worseck, G., \& Wisotzki, L. 2006, A\&A, 450, 495

Yu, Q., \& Tremaine, S. 2002, MNRAS, 335, 965 